Pacific

Journal of

Mathematics

MOTION OF HYPERSURFACES BY GAUSS CURVATURE Ben Andrews 


\title{
MOTION OF HYPERSURFACES BY GAUSS CURVATURE
}

\section{BEN ANDREWS}

\begin{abstract}
We consider $\boldsymbol{n}$-dimensional convex Euclidean hypersurfaces moving with normal velocity proportional to a positive power $\alpha$ of the Gauss curvature. We prove that hypersurfaces contract to points in finite time, and for $\alpha \in(1 /(n+2], 1 / n]$ we also prove that in the limit the solutions evolve purely by homothetic contraction to the final point. We prove existence and uniqueness of solutions for non-smooth initial hypersurfaces, and develop upper and lower bounds on the speed and the curvature independent of initial conditions. Applications are given to the flow by affine normal and to the existence of non-spherical homothetically contracting solutions.
\end{abstract}

\section{Introduction.}

Motivation for the study of hypersurfaces moving by their Gauss curvature comes from several sources:

1.1. Tumbling stones. W.J. Firey introduced the Gauss curvature flow in 1974, as a model of the wearing process undergone by a pebble on a beach $[\mathbf{F i}]$. Consider a stone which occupies an open, bounded convex region of $\mathbb{R}^{n+1}$ at time $t=0$. The stone tumbles, and collides with a hyperplane (the beach) with random orientation. We assume for simplicity that the amount of material removed in a collision at a point $x$ of the stone depends only on the normal direction $\nu_{x}$ (thus allowing some anisotropy in the material of the stone). The number of collisions with a region $B$ of the surface of the stone is proportional to the measure of the set $\nu(B)=\left\{\nu_{x}: x \in B\right\} \subset S^{n}$ of normal directions to $B$. This is equal to $\int_{B} K_{x} d \mathcal{H}^{n}(x)$, where $K_{x}$ is the Gauss curvature of the hypersurface at $x$. The rate at which the stone wears away at a point $x$ is given by $\rho\left(\nu_{x}\right) K_{x}$ for some positive function $\rho$ on $S^{n}$, and we have the evolution equation

$$
\dot{x}=-\rho\left(\nu_{x}\right) K_{x} \nu_{x} .
$$

\subsection{Affine geometry: Inner parallel surfaces and the affine normal} flow. K. Leichtweiss introduced the notion of inner parallel surfaces for a convex body in affine geometry in the paper $[\mathbf{L e}]$. Given a convex region $\Omega$, the idea is to construct a family of related regions $P_{t} \Omega$, by a procedure which is well-defined in the setting of affine geometry — that is, if we perform 
an area-preserving affine transformation $L$ to get a new region $L \Omega$, then $P_{t}(L \Omega)=L\left(P_{t} \Omega\right)$.

The procedure is as follows: For each direction $z \in S^{n}$, there exists a unique supporting hyperplane $H_{z}=\{\langle z, y\rangle=h(z)\}$ to $\Omega$ with normal direction $z$ pointing outward from $\Omega$. The notion of parallel hyperplanes is well-defined in affine geometry, as is the notion of the volume of a region. Hence we can choose a unique hyperplane $H_{z, t}$ parallel to $H_{z}$ such that the volume of the part of $\Omega$ between $H_{z, t}$ and $H_{z}$ is equal to $t^{(n+2) / 2}$ (in the case where $\Omega$ is the region above a paraboloid, this choice of exponent ensures that $H_{z, t}$ moves at constant speed). Equivalently, we can define a function $h_{t}(z)$ by the requirement

$$
\operatorname{Vol}\left(\left\{y \in \Omega: h_{t}(z) \leq\langle y, z\rangle \leq h(z)\right\}\right)=t^{(n+2) / 2} .
$$

Then we define $P_{t} \Omega$ to be the convex set

$$
\bigcap_{z \in S^{n}}\left\{y \in \mathbb{R}^{n+1}:\langle z, y\rangle \leq h_{t}(z)\right\} .
$$

In contrast to the corresponding situation in Euclidean geometry, this procedure does not define a semi-group: If we begin with a region $\Omega$, construct the regions $P_{t} \Omega$, and use them to construct the regions $P_{\tau} P_{t} \Omega$, then these are not in general given by $P_{t^{\prime}} \Omega$ for any $t^{\prime}$. To remedy this we consider $P_{t / n}^{n} \Omega$, obtained by following the above construction repeatedly over small intervals, and take the limit $n \rightarrow \infty$ of infinitesimally small steps to obtain a region $\tilde{P}_{t} \Omega$. This defines a deformation which is clearly well-defined in affine geometry, and satisfies the semi-group property $\tilde{P}_{t} \tilde{P}_{\tau}=\tilde{P}_{t+\tau}$.

To find an explicit description of this deformation in the case where $\Omega$ is smooth and strictly convex, we consider the regions $P_{t} \Omega$ in the limit of small $t$ : Fix $z$, and choose coordinates for $\mathbb{R}^{n+1}$ such that $e_{1}, \ldots, e_{n}$ span the supporting hyperplane $H_{z}$ of $\Omega$, and the supporting point is at the origin. Then $M=\partial \Omega$ is locally a graph in these coordinates:

$$
x_{n+1}=-\frac{1}{2} \sum_{i, j=1}^{n} h_{i j} x_{i} x_{j}+O\left(|x|^{3}\right),
$$

where $h_{i j}$ is the second fundamental form at the supporting point. There exists a volume-preserving linear transformation which fixes the $e_{n+1}$ direction and brings $M$ locally to the form

$$
x_{n+1}=-\frac{1}{2} K^{1 / n} \sum_{i=1}^{n} x_{i}^{2}+O\left(|x|^{3}\right)
$$

where $K=\operatorname{det} h_{i j}$ is the Gauss curvature at the supporting point. Then

$$
\begin{aligned}
& \operatorname{Vol}(\{y \in \Omega: h(z)-d \leq\langle y, z\rangle \leq h(z)\}) \\
& =2^{n / 2} \omega_{n} K^{-1 / 2} d^{(n+2) / 2}+O\left(d^{(n+3) / 2}\right)
\end{aligned}
$$


where $\omega_{n}$ is the volume of the unit ball in $\mathbb{R}^{n}$, and hence the requirement (2) implies that

$$
h_{t}(z)=h(z)-\frac{K^{\frac{1}{n+2}}}{2^{\frac{n}{n+2}} \omega_{n}^{\frac{2}{n+2}}} t+O\left(t^{3 / 2}\right) .
$$

It follows that the limiting deformation is given by the equation

$$
\dot{x}=-c_{n} K_{x}^{\frac{1}{n+2}} \nu_{x} .
$$

This evolution equation is the simplest invariant flow in affine differential geometry; up to reparametrisation it is the motion of a hypersurface in the direction of its affine normal vector. This has been considered in [ST1, ST2] for the case of convex curves in the plane, and in [A4] more generally. For nonconvex curves results were recently obtained in $[\mathbf{A S T}]$.

1.3. Image analysis. Many fundamental problems in image analysis have been approached using geometric flows: An image represented by a greyscale density function $u$ can be processed to remove noise by smoothing the level sets of $u$ with a parabolic flow. Various candidates have been considered, but in [AGLM] axioms were proposed which included the natural requirement of affine invariance. This leads to the evolution Equation (4). In the case of nonconvex hypersurfaces this is no longer parabolic, and various authors (see $[\mathbf{A G L M}],[\mathbf{C S}],[\mathbf{N K}]$ ) have considered the generalization (for two-dimensional surfaces)

$$
\dot{x}=-(\operatorname{sgn} H) \max \{K, 0\}^{1 / 4} \nu
$$

where $H$ is the mean curvature. Applications of plane curve evolution equations to image analysis and computer vision are described in [AGLM], [OST], and [ST1]-[ST3].

1.4. Gradient flows of the mean width. The width of a convex region $\Omega$ in a direction $z \in S^{n}$ is defined by

$$
w(z)=\sup _{y_{1}, y_{2} \in \Omega}\left\langle y_{1}-y_{2}, z\right\rangle=h(z)+h(-z)
$$

where $h(z)=\sup _{y \in \Omega}\langle y, z\rangle$ is the support function of $\Omega$. The mean width $V_{1}(\Omega, \varphi)$ with respect to a measure $\varphi d \mu$ on $S^{n}$ is given (up to a constant factor) by integrating the width over all directions $z \in S^{n}$ :

$$
\begin{aligned}
V_{1}(\Omega)=\int_{S^{n}} w(z) \varphi(z) d \mu(z) & =\int_{S^{n}} h(z)(\varphi(z)+\varphi(-z)) d \mu(z) \\
& =\int_{\partial \Omega} K h \tilde{\varphi} d \mathcal{H}^{n}
\end{aligned}
$$


where $\tilde{\varphi}(z)=\varphi(z)+\varphi(-z)$. The first variation formula for the mean width can be calculated as follows: Consider a smooth family $\Omega_{t}$ of convex regions, with support functions $h_{t}(z)$ such that

$$
\left.\frac{\partial}{\partial t} h_{t}(z)\right|_{t=0}=f(z)
$$

then

$$
\left.\frac{d}{d t} V_{1}\left(\Omega_{t}\right)\right|_{t=0}=\int_{S^{n}} \tilde{\varphi}(z) f(z) d \mu=\int_{\partial \Omega_{0}} K f \tilde{\varphi} d \mathcal{H}^{n} .
$$

We consider the flow of steepest descent of the mean width in $L^{p}$ spaces on $\partial \Omega$ - that is, we seek that variation $f$ for which $V_{1}\left(\Omega_{t}, \varphi\right)$ decreases fastest amongst all variations with the same $L^{p} \operatorname{norm}\left(\int_{\partial \Omega}|f|^{p} \sigma(\nu) d \mathcal{H}^{n}\right)^{1 / p}$ ( $\sigma$ is a positive smooth function on $S^{n}$ ): By the Hölder inequality we have for $p>1$

$$
\begin{aligned}
\left|\int_{\partial \Omega_{0}} K f \tilde{\varphi} d \mathcal{H}^{n}\right| & =\left|\int_{\partial \Omega_{0}} K f \frac{\tilde{\varphi}}{\sigma} \sigma d \mathcal{H}^{n}\right| \\
& \leq\left(\int_{\partial \Omega_{0}}|f|^{p} \sigma d \mathcal{H}^{n}\right)^{1 / p}\left(\int_{\partial \Omega_{0}}\left(\frac{K \tilde{\varphi}}{\sigma}\right)^{\frac{p}{p-1}} \sigma d \mathcal{H}^{n}\right)^{1-1 / p}
\end{aligned}
$$

with equality if and only if $f=c(K \tilde{\varphi} / \sigma)^{1 /(p-1)}$. The flow of steepest descent is therefore

$$
\dot{x}=-\rho\left(\nu_{x}\right) K_{x}^{1 /(p-1)} \nu_{x}
$$

where $\rho=(\tilde{\varphi} / \sigma)^{1 /(p-1)}$ is a smooth positive function on $S^{n}$.

\subsection{Evolving hypersurfaces and degenerate fully nonlinear PDE.}

The evolution equations derived above are included in a large class of parabolic evolution equations for hypersurfaces which have been considered before. Simplest in this class is the mean curvature flow, in which a hypersurface moves in the direction of its inward normal with speed given by the mean curvature. Huisken $[\mathbf{H u}]$ showed that convex hypersurfaces moving under such equations contract to points in finite time, and that the hypersurfaces become spherical in shape in the process. This argument has since been extended to many processes where convex hypersurfaces move with speeds given by homogeneous degree one, concave or convex monotone symmetric functions of the principal curvatures: Chow considered flows by the $n$th root of the Gauss curvature [Ch1] and the square root of the scalar curvature [Ch2], and the author has considered a general class of such evolution equations [A1]. Corresponding results for flows where the speed has other positive degrees of homogeneity in the curvature seem much harder to prove. The author has treated the special case of flow by the power $1 /(n+2)$ of the Gauss curvature, which is the flow by affine normal [A4]. Tso [Ts] 
and Chow [Ch1] have shown that hypersurfaces moving with speed equal to any positive power of the Gauss curvature contract to points in finite time.

The Gauss curvature flows form a convenient class of examples of parabolic equations with varying degeneracy: For large $\alpha$ they become more degenerate, and for small $\alpha$ they become singularly parabolic. Intermediate values of $\alpha$ are singular in some situations and degenerate in others. The precise effect of such degeneracy or singularity on the regularity of solutions is extremely complicated. In particular, it would be interesting to know how irregular solutions can be, how the regularity estimates depend on time (particularly where the initial solution is highly irregular), and how solutions behave in the neighbourhood of degenerate or singular regions.

There are several other important families of PDE for which similar questions can be asked - in particular, natural families of parabolic equations with varying degeneracy include the porous medium equations

$$
\dot{u}=\Delta\left(|u|^{m-1} u\right),
$$

and the $p$-harmonic heat flows

$$
\dot{u}=\nabla \cdot\left(|\nabla u|^{p-2} \nabla u\right),
$$

for which there is also a natural generalization to $p$-harmonic maps between Riemannian manifolds. The Gauss curvature flows can be considered a geometric analogue of the porous medium equations.

In the case of curves in the plane, more complete results are known: Gage [Ga1]-[Ga2] and Hamilton [GH] showed that convex curves contract to points in finite time and become round under the curve shortening flow (where the speed of motion equals the curvature), and Grayson $[\mathbf{G r}]$ extended this by showing that any embedded curve eventually becomes convex. This was extended to include anisotropic analogues of the curve-shortening flow by Gage [Ga3] in the convex case, and by Oaks [Oa] for nonconvex curves. The author considered equations of varying degeneracy in the convex case [A2], [A8], and obtained optimal estimates on the regularity of solutions, including their initial behaviour. The particular case of the affine normal flow has also been extended to nonconvex curves $[\mathbf{A S T}]$.

\section{The result.}

Our main aim in this paper is to prove results about the regularity and limiting behaviour of solutions of the Gauss curvature flows of the form

$$
\frac{d x}{d t}=-\rho(\nu(x)) K(x)^{\alpha} \nu(x),
$$

where $\alpha$ is in the range $(1 /(n+2), 1 / n]$. These particular exponents arise as follows in the proof: We first prove (in Section 4 of the paper) that the solutions of Gauss curvature flows have isoperimetric ratio bounded as long 
as they exist, provided $\alpha$ is greater than the critical value $1 /(n+2)$. This value is sharp - the flow with $\rho \equiv 1$ and $\alpha=1 /(n+2)$ is the affine normal flow, for which solutions converge to ellipsoids of arbitrary eccentricity [A4], and so the isoperimetric ratio tends to stay bounded but does not generally improve; in a separate paper [A9] we prove that for exponents smaller than this (or equal to this if $\rho$ is nonconstant) there are solutions which have isoperimetric ratios approaching infinity. Second, we prove (in Sections 5 and 6) that if the hypersurface has bounded isoperimetric ratio, and $\alpha \leq$ $1 / n$, then a short time later the moving hypersurfaces are strictly convex and have bounded curvature. The exponent $1 / n$ is again sharp, as there are solutions of the Gauss curvature flow for any $\alpha>1 / n$ which remain nonstrictly convex and are not $C^{\infty}$ — in fact any initial convex hypersurface which includes a planar piece will behave this way. This phenomenon was first noted by Richard Hamilton for the case $\alpha=1$ [Ha1]. We describe such behaviour more fully in Section 12 of this paper.

By combining these results, we obtain the following:

Theorem 1. For any open bounded convex region $\Omega_{0}$, any smooth positive function $\rho: S^{n} \rightarrow \mathbb{R}$, and any $\alpha \in(1 /(n+2), 1 / n]$, there exists a family of embeddings $x: S^{n} \times[0, T) \rightarrow \mathbb{R}^{n+1}$ satisfying (6), unique up to composition with an arbitrary time-independent diffeomorphism, such that $M_{t}=x\left(S^{n}, t\right)$ converges in Hausdorff distance to the boundary of the region $\Omega_{0}$ as $t$ approaches zero. $x$ is smooth and strictly convex for $t>0$ and converges to a point $p \in \mathbb{R}^{n+1}$ as $t$ approaches $T$. Furthermore, the hypersurfaces

$$
\tilde{M}_{t}=\left(\frac{\operatorname{Vol}\left(S^{n}\right)}{\operatorname{Vol}\left(M_{t}\right)}\right)^{1 /(n+1)}\left(M_{t}-p\right)
$$

converge in $C^{\infty}$ as $t$ approaches $T$, to a smooth, strictly convex limit hypersurface $\tilde{M}_{T}$ for which $\langle x, \nu\rangle=c \rho(\nu) K^{\alpha}$ for some $c>0$.

This means that the evolving hypersurface contracts to a point, and asymptotically approaches a solution which evolves purely by homothetically scaling about this limiting point.

We also have the following generalisation for smaller $\alpha$ :

Theorem 2. The result of Theorem 1 also holds for a solution of (6) with any $\alpha \in(0,1 / n]$, provided the isoperimetric ratios of the evolving hypersurfaces remain bounded.

We deduce in Section 7 the first part of Theorem 1, that solutions contract to points in finite time (in fact we prove this for any $\alpha>0$ ). This was proved previously for isotropic cases by Tso [Ts] and Chow [Ch1]. In Section 8 we prove that solutions exist starting from singular initial hypersurfaces, and immediately become smooth and strictly convex if $\alpha \leq 1 / n$ - note that we make no regularity assumptions about the initial hypersurface, other than 
those implied by its convexity. We also prove in Theorem 15 the existence of unique viscosity solutions for $\alpha>1 / n$, although this is not required for the proof of Theorems 1 and 2. In Section 9 we digress from the main argument of the paper to apply the regularity and convexity estimates in a simple new proof of the convergence theorem for the affine normal flow. In Section 11 of the paper we use Theorem 2 to deduce the existence of non-spherical homothetic solutions of Equation (6) for constant $\rho$ and suitable $\alpha$ between 0 and $1 /(n+2)$.

We remark that Urbas [U2] has considered noncompact solutions of isotropic equations of the form (6), in particular proving the existence of solutions which evolve by homothetically expanding or translating.

\section{Notation and preliminaries.}

The inradius $r_{-}$of an open convex region is the supremum of the radii of all balls contained in it, and the circumradius $r_{+}$is the infinum of the radii of balls containing it. In this paper we refer to the ratio $r_{-} / r_{+}$as the isoperimetric ratio of the body.

For a convex region with boundary given by a smooth embedding $x: M \rightarrow$ $\mathbb{R}^{n+1}$, we have an outward unit normal vector field $\nu: M \rightarrow S^{n} \subset \mathbb{R}^{n+1}$, which we use to define the Weingarten map $\mathcal{W}_{x}: T_{x} M \rightarrow T_{x} M$ by the formula

$$
\mathcal{W}(u)=D_{u} \nu \in T_{\nu(x)} S^{n} \simeq T_{x} M
$$

for any $x \in M$ and $u \in T_{x} M$. The eigenvalues $\lambda_{1}, \ldots, \lambda_{n}$ of $\mathcal{W}(x)$ are the principal curvatures of $M$ at $x$. The elementary symmetric functions $E_{j}$ of these are defined by

$$
E_{j}=\frac{1}{\left(\begin{array}{l}
n \\
k
\end{array}\right)} \sum_{1 \leq i_{1}<i_{2}<\cdots<i_{j} \leq n}\left(\prod_{k=1}^{j} \lambda_{i_{k}}\right) .
$$

In particular, $K=E_{n}$ is the Gauss curvature, and $H=E_{1}$ is the mean curvature.

The covariant derivative $\nabla$ on the hypersurface is given by the formula

$$
\nabla_{u} v=D_{u} v+\langle\mathcal{W}(u), v\rangle \nu
$$

We will find it convenient at some points in this paper to describe an open convex region $\Omega \subset \mathbb{R}^{n+1}$ in terms of its support function $h: S^{n} \rightarrow \mathbb{R}$, defined by

$$
h(z)=\sup _{x \in \Omega}\langle x, z\rangle .
$$

The support function completely describes the region $\Omega$ - in particular, $\Omega$ can be recovered from $h$ via the expression

$$
\Omega=\cap_{z \in S^{n}}\left\{y \in \mathbb{R}^{n+1}:\langle z, y\rangle<h(z)\right\} .
$$


Alternatively, in the case where $M$ is strictly convex and smooth, the support function can be used to define a canonical embedding $\bar{x}$ of $S^{n}$ with image equal to $M$ :

$$
\bar{x}(z)=h(z) z+\bar{\nabla}_{i} h(z) \bar{g}^{i j} \bar{\nabla}_{j} z .
$$

This has the property that the outward normalto $M$ at the point $\bar{x}(z)$ is equal to $z$, for each $z \in S^{n}$.

The Weingarten map can also be recovered directly from $h$ :

$$
\left\langle\mathcal{W}^{-1}(u), v\right\rangle=\bar{\nabla}_{u} \bar{\nabla}_{v} h+\langle u, v\rangle h
$$

where $\bar{\nabla}$ is the covariant derivative on $S^{n}$, and we identify $T_{x} M$ and $T_{\nu(x)} S^{n}$. For convenience we will denote by $\mathfrak{r}_{i j}$ the corresponding symmetric bilinear form, the eigenvalues of which are the principal radii of curvature $r_{i}=\lambda_{i}^{-1}$, $i=1, \ldots, n$ :

$$
\mathfrak{r}_{i j}=\bar{\nabla}_{i} \bar{\nabla}_{j} h+\bar{g}_{i j} h .
$$

$\mathfrak{r}_{i j}$ satisfies a Codazzi-type identity:

$$
\begin{aligned}
\bar{\nabla}_{k} \mathfrak{r}_{i j} & =\bar{\nabla}_{k} \bar{\nabla}_{i} \bar{\nabla}_{j} h+\bar{g}_{i j} \bar{\nabla}_{k} h \\
& =\bar{\nabla}_{i} \bar{\nabla}_{k} \bar{\nabla}_{j} h+\left(\bar{g}_{k j} \bar{\nabla}_{i} h-\bar{g}_{i j} \bar{\nabla}_{k} h\right)+\bar{g}_{i j} \bar{\nabla}_{k} h \\
& =\bar{\nabla}_{i} \mathfrak{r}_{k j} .
\end{aligned}
$$

Differentiating (11), commuting derivatives, and applying (11) again to the result, we obtain a version of the Simons' identity for the second derivatives of the second fundamental form $[\mathbf{S i}]$ :

$$
\bar{\nabla}_{(i} \bar{\nabla}_{j)} \mathfrak{r}_{k l}=\bar{\nabla}_{(k} \bar{\nabla}_{l)} \mathfrak{r}_{i j}+\bar{g}_{i j} \mathfrak{r}_{k l}-\bar{g}_{k l} \mathfrak{r}_{i j}
$$

where the brackets denote symmetrisation.

For convenience, we will denote by $S_{k}$ the $k$ th elementary symmetric function of the eigenvalues of $\mathfrak{r}_{i j}$. In particular, $S_{n}=K^{-1} . S_{k}$ may be considered as a function of the components of the matrix $\mathfrak{r}_{i j}$, and we denote by $\dot{S}_{k}^{i j}$ and $\ddot{S}_{k}^{i j p q}$ the first and second derivatives:

$$
\dot{S}_{k}^{i j}=\frac{\partial S_{k}}{\partial \mathfrak{r}_{i j}}
$$

and

$$
\ddot{S}_{k}^{i j p q}=\frac{\partial^{2} S_{k}}{\partial \mathfrak{r}_{i j} \partial \mathfrak{r}_{p q}}
$$

$\dot{S}_{k}$ is a positive definite symmetric bilinear form provided $\mathfrak{r}$ is positive definite, and $S_{k}^{1 / k}$ is a concave function of the components of $\mathfrak{r}$ for $k=1, \ldots, n$ [Mi]. 
The support function allows the degenerate parabolic system of equation (6) to be re-written as a parabolic scalar equation (see [U1], [A1], [A4]):

$$
\frac{d h(z)}{d t}=-\rho(z) \operatorname{det}\left(\bar{\nabla}^{2} h+\operatorname{Id} h\right)^{-\alpha} .
$$

In particular, this implies the existence of a smooth solution of Equation (6) for a short time for any smooth, strictly convex initial hypersurface.

In a region where a family of hypersurfaces moving under Equation (6) can be represented as graphs $x_{n+1}=u_{t}\left(x_{1}, \ldots, x_{n}\right)$ for some convex functions $u_{t}$, we can work with an equivalent scalar parabolic equation for the functions $u_{t}$ :

$$
\frac{\partial}{\partial t} u=\tilde{\rho}(D u) \frac{\left(\operatorname{det} D^{2} u\right)^{\alpha}}{\left(1+|D u|^{2}\right)^{\frac{\alpha(n+2)-1}{2}}}
$$

where

$$
\tilde{\rho}(D u)=\rho\left(\frac{\sum_{i=1}^{n} e_{i} D_{i} u-e_{n+1}}{\sqrt{1+|D u|^{2}}}\right) .
$$

We note some elementary features of Eq. (6): First, the speed of motion is given by a homogeneous function of the curvatures, and this homogeneity leads to a scaling property of solutions. Specifically, if $x: M \times[0, T] \rightarrow \mathbb{R}^{n+1}$ satisfies Eq. (6), then for each $\lambda>0$ another solution $x_{\lambda}: M \times\left[0, \lambda^{1+n \alpha} T\right] \rightarrow$ $\mathbb{R}^{n+1}$ is given by

$$
x_{\lambda}(p, t)=\lambda x\left(p, \lambda^{-(1+n \alpha)} t\right) .
$$

This also implies corresponding scaling invariance properties for the solutions of Equations (13) and (14).

A second important property of solutions of Eq. (6) is the comparison principle: If $\left\{M_{t}^{(i)}\right\}, i=1,2$ are two families of smooth, strictly convex hypersurfaces moving under Eq. (6), and $M_{0}^{(1)} \cap M_{0}^{(2)}=\emptyset$, then $M_{t}^{(1)} \cap M_{t}^{(2)}=\emptyset$ for all $t>0$ in the common interval of existence. A local version also holds: If $\left\{M_{t}^{(i)}\right\}, i=1,2$ are families of smooth, strictly convex hypersurfaces with boundary, $M_{0}^{(1)} \cap M_{0}^{(2)}=\emptyset$, and $M_{t}^{(1)} \cap \partial M_{t}(2)=M_{t}^{(2)} \cap \partial M_{t}(1)=\emptyset$ for $t \in[0, T]$, then $M_{t}^{(1)} \cap M_{t}^{(2)}=\emptyset$ for $t \in[0, T]$.

\section{Monotone quantities and diameter bounds.}

In this section we prove that whenever $\alpha>1 /(n+2)$, the evolving hypersurfaces have bounded isoperimetric ratios for as long as the solution exists. The main tool used here is an integral estimate known as the entropy estimate, which was proved for the case $\alpha=1$ by Chow [Ch1], and for other $\alpha$ by the author $[\mathbf{A} \mathbf{3}]$. 
We define an integral quantity $\mathcal{Z}_{\rho, \alpha}$ for any given $\alpha$ and $\rho$ by

$$
\mathcal{Z}_{\rho, \alpha}=\operatorname{Vol}(M)^{n /(n+1)}\left(\frac{1}{\int_{S^{n}} \rho d \mu} \int_{M} \rho K^{\alpha} d \mathcal{H}^{n}\right)^{1 /(\alpha-1)}
$$

if $\alpha \neq 1$, and

$$
\mathcal{Z}_{\rho, 1}=\operatorname{Vol}(M)^{n /(n+1)} \exp \left\{\frac{1}{\int_{S^{n}} \rho d \mu} \int_{M} \rho K \log K d \mathcal{H}^{n}\right\}
$$

if $\alpha=1$. For convenience, we also denote by $\mathcal{Z}_{\natural, \alpha}$ the same quantity with $\rho \equiv 1$.

Theorem 3. For any smooth, strictly convex solution of Equation (6),

$$
\frac{d}{d t} \mathcal{Z}_{\rho, \alpha} \leq 0
$$

with equality if and only if the equation $\langle x, \nu\rangle=c \rho(\nu) K^{\alpha}$ holds for some $c>0$ and some choice of origin in $\mathbb{R}^{n+1}$.

This integral bound will be combined with the following estimate to deduce isoperimetric ratio bounds for solutions of the flow:

Theorem 4. For any smooth, strictly convex hypersurface $M^{n}$ in $\mathbb{R}^{n+1}$,

$$
\frac{r_{+}(M)}{r_{-}(M)} \leq C(\alpha, \rho) \mathcal{Z}_{\rho, \alpha}^{(n+1) \beta(\alpha)}
$$

for some positive constant $\beta(\alpha)$, provided $\alpha>1 /(n+2)$.

Proof. We begin with a bound in terms of $\mathcal{Z}_{\natural, \alpha}$ :

Following [Ha2], we begin by obtaining a lower bound on the $n$-dimensional areas of projections of $M$ onto hyperplanes: Given a direction $z_{0} \in S^{n}$, the area of the projection on to the plane with normal $z_{0}$ is given by

$$
A_{z_{0}}=\frac{1}{2} \int_{S^{n}}\left|\left\langle z, z_{0}\right\rangle\right| S_{n} d \mu .
$$

We apply the Hölder inequality to bound this from below, as follows:

$$
\begin{aligned}
A_{z_{0}} & =\frac{1}{2} \int_{S^{n}}\left|\left\langle z, z_{0}\right\rangle\right| S_{n} d \mu \\
& \geq \frac{1}{2}\left(\int_{S^{n}} S_{n}^{1-\alpha} d \mu\right)^{1 /(1-\alpha)}\left(\int_{S^{n}}\left|\left\langle z, z_{0}\right\rangle\right|^{1-1 / \alpha} d \mu\right)^{\alpha /(\alpha-1)}
\end{aligned}
$$

provided $\alpha \in(0,1)$. The integral $\int_{S^{n}}\left|\left\langle z, z_{0}\right\rangle\right|^{\beta} d \mu$ is bounded for $\beta>-1$. Hence for $\alpha \in(1 / 2,1)$ we have

$$
A_{z_{0}} \geq C\left(\int_{S^{n}} S_{n}^{1-\alpha} d \mu\right)^{1 /(1-\alpha)}=C V^{n /(n+1)} \mathcal{Z}_{\natural, \alpha}^{-1} .
$$

For $\alpha \geq 1$ this inequality still holds, because by the Hölder inequality $\mathcal{Z}_{\natural, \alpha}$ is increasing in $\alpha$. 
Finally, we consider the case where $\alpha \in(1 /(n+2), 1 / 2]$ : The Hölder inequality gives

$$
\mathcal{Z}_{\natural, \alpha} \geq \mathcal{Z}_{\natural, 1 /(n+2)}^{\frac{(n+1)(3 / 4-\alpha)}{4(1-\alpha)(3 n+2)}} \mathcal{Z}_{\natural, 3 / 4}^{\frac{\alpha-1 /(n+2)}{(1-\alpha)(3-4 /(n+2))}}
$$

The affine isoperimetric inequality (see $[\mathbf{B}], \S 26$ and $\S 73$, [Sa] or $[\mathbf{A 4}]$, Theorem 7.1) implies that $\mathcal{Z}_{\natural, 1 /(n+2)} \geq 1$. Hence by applying the bound on $A_{z_{0}}$ in terms of $\mathcal{Z}_{\natural, 3 / 4}$, which we know from the cases treated above, we have

$$
A_{z_{0}} \geq C V^{n /(n+1)} \mathcal{Z}_{\natural, 3 / 4}^{-1} \geq C V^{n /(n+1)} \mathcal{Z}_{\natural, \alpha}^{-\frac{(1-\alpha)(3-4 /(n+2))}{\alpha-1 /(n+2)}} .
$$

Hence for each $\alpha \in(1 /(n+2), \infty)$ we have $A_{z_{0}} \geq C \mathcal{Z}_{\natural, \alpha}^{-\beta(\alpha)}$ for some constant $C$ and some positive exponent $\beta(\alpha)$.

Next we deduce a bound on the maximum width of $M$ (the largest distance between parallel supporting hyperp lanes): Let $z_{0}$ be the normal direction of a pair of parallel supporting hyperplanes for $M$ at maximal separation. Then the points of contact of $M$ with these two planes are joined by a segment with length equal to the maximum width of $M$, and which is entirely contained in $M$. Choosing the origin to be at the centre of this segment, we have $h\left(z_{0}\right)=h\left(-z_{0}\right)=w_{+} / 2$ where $w_{+}$is the maximum width of $M$, and $h(z) \geq\left|\left\langle z, z_{0}\right\rangle\right| h\left(z_{0}\right)$ for all $z \in S^{n}$. But then the enclosed volume of $M$ is computed by:

$\operatorname{Vol}(M)=\frac{1}{n+1} \int_{S^{n}} h S_{n} d \mu \geq \frac{1}{n+1} \int_{S^{n}} h\left(z_{0}\right)\left|\left\langle z, z_{0}\right\rangle\right| S_{n} d \mu=\frac{w_{+}}{2(n+1)} A_{z_{0}}$.

Hence $w_{+} \leq 2(n+1) \operatorname{Vol}(M) / A_{z_{0}} \leq C \mathcal{Z}_{\natural, \alpha}^{\beta(\alpha)} \operatorname{Vol}(M)^{1 /(n+1)}$.

Note that $\operatorname{Vol}(M) \leq w_{-} w_{+}^{n}$ where $w_{-}$is the minimum width of $M$, since $M$ in contained between $(n+1)$ pairs of parallel planes in any set of orthonormal directions; and, in particular, in the case where one of the pairs of planes is at minimal separation. Then the separation of all the other pairs is bounded by $w_{+}$. It follows that $w_{-} \geq V w_{+}^{-n} \geq C \mathcal{Z}_{\natural, \alpha}^{-n \beta(\alpha)} \operatorname{Vol}(M)^{1 /(n+1)}$.

This gives a bound on the ratio of the minimum and maximum widths of the hypersurface, and this is sufficient to bound the isoperimetric ratio (see for example [A1], Lemma 5.4).

Finally, we consider the anisotropic cases $\rho \neq$ const.: For $\alpha<1, \mathcal{Z}_{\rho, \alpha}$ is comparable to $\mathcal{Z}_{\natural, \alpha}$ :

$$
\frac{\inf _{S^{n}} \rho^{1 /(1-\alpha)}}{\int_{S^{n}} \rho} \mathcal{Z}_{\natural, \alpha} \leq \mathcal{Z}_{\rho, \alpha} \leq \frac{\sup _{S^{n}} \rho^{1 /(1-\alpha)}}{\int_{S^{n}} \rho} \mathcal{Z}_{\natural, \alpha} .
$$

For $\alpha \geq 1$ the desired inequality results from the monotonicity of $\mathcal{Z}_{\rho, \alpha}$ as a function of $\alpha$, a consequence of the Hölder inequality. 
We have shown in particular that for $\alpha>1 /(n+2)$, any solution of Equation (6) with smooth, strictly convex initial data has uniformly bounded isoperimetric ratio on the entire interval of its existence.

\section{Displacement and speed bounds.}

In this section we prove that the ratio of the maximum and minimum values of the speed remains uniformly bounded for as long as the solution exists.

We will first deduce upper bounds on the displacement of the hypersurfaces, by using spheres enclosed within $M_{0}$ as barriers:

Theorem 5. For any $\alpha>0$ and smooth positive $\rho$, and any smooth, strictly convex solution $\left\{M_{t}\right\}_{t>0}$ of Eq. (13),

$$
h(z, t) \geq h(z, 0)-C \frac{r_{+}\left(M_{0}\right)}{r_{-}\left(M_{0}\right)} t^{\frac{1}{1+n \alpha}}
$$

for all $t \in\left(0, C^{\prime} r_{-}\left(M_{0}\right)^{1+n \alpha}\right]$ in the interval of existence of the solution, where $C$ and $C^{\prime}$ depend only on $\alpha$ and $\rho$.

Proof. Choose the origin at the centre of a ball of radius $r_{-}\left(M_{0}\right)$ enclosed by $M_{0}$. Fix $z \in S^{n}$, and define for each $\varepsilon \in(0,1]$ a sphere

$$
S_{\varepsilon}=\left\{y \in \mathbb{R}^{n+1}:|y-(1-\varepsilon) \bar{x}(z)|=\varepsilon r_{-}\left(M_{0}\right)\right\} .
$$

Then $S_{\varepsilon}$ is contained in the convex hull of $\bar{x}(z)$ and $B_{r_{-}\left(M_{0}\right)}(0)$, so by convexity is contained in $M_{0}$.

Any family of spheres of the form $S_{r(t)}(p)$ with $p \in \mathbb{R}^{n+1}$ and

$$
r(t)=\left(r(0)^{1+n \alpha}-\sup \rho(1+n \alpha) t\right)^{1 /(1+n \alpha)}
$$

satisfies $\langle\dot{x}, \nu\rangle \leq-\rho K^{\alpha}$, and hence act as barriers for solutions of Eq. (6), by the comparison principle.

This gives an estimate on the support function of $M_{t}$ in direction $z: S_{\varepsilon}$ produces a barrier which shrinks to its centre at time $\varepsilon^{1+n \alpha} r_{-}(M)^{1+n \alpha} /((1+$ $n \alpha) \sup \rho$ ), and we have

$$
h(z, t)-h(z, 0) \geq-\frac{((1+n \alpha) \sup \rho t)^{\frac{1}{1+n \alpha}}}{r_{-}(M)} h(z, 0) \geq-C(\alpha, \rho) \frac{r_{+}\left(M_{0}\right)}{r_{-}\left(M_{0}\right)} t^{\frac{1}{1+n \alpha}} .
$$

Our next estimate is a speed bound, which we prove using the maximum principle applied to the evolution equations for the speed and the support function. The proof is related to that given in [Ts] for the isotropic case of Eq. (1). 
Theorem 6. For any smooth, strictly convex solution $\left\{M_{t}\right\}_{[0, T]}$ of Eq. (13) with $R_{-} \leq r_{-}\left(M_{t}\right) \leq r_{+}\left(M_{t}\right) \leq R_{+}$for $t \in[0, T]$,

$$
\rho(z) S_{n}^{-\alpha} \leq C(n, \alpha, \rho)\left(R_{-}^{-n \alpha}+\left(\frac{R_{+}}{R_{-} t}\right)^{\frac{n \alpha}{1+n \alpha}}\right) .
$$

Proof. From the definition (10) and the evolution Equation (13) we obtain

$$
\frac{\partial}{\partial t} \mathfrak{r}_{i j}=-\left(\bar{\nabla}_{i} \bar{\nabla}_{j}\left(\rho S_{n}^{-\alpha}\right)+\bar{g}_{i j} \rho S_{n}^{-\alpha}\right) .
$$

Since $S_{n}=\operatorname{det} \mathfrak{r}_{i j}$, this implies

$$
\frac{\partial}{\partial t}\left(\rho S_{n}^{-\alpha}\right)=\alpha \rho S_{n}^{-(1+\alpha)} \dot{S}_{n}^{k l}\left(\bar{\nabla}_{k} \bar{\nabla}_{l}\left(\rho S_{n}^{-\alpha}\right)+\bar{g}_{k l} \rho S_{n}^{-\alpha}\right) .
$$

We also have

$$
\begin{aligned}
\frac{\partial}{\partial t} h(z, t) & =-\rho S_{n}^{-\alpha} \\
& =\alpha \rho S_{n}^{-(1+\alpha)} \dot{S}_{n}^{k l}\left(\bar{\nabla}_{k} \bar{\nabla}_{l} h+\bar{g}_{k l} h\right)-(1+n \alpha) \rho S_{n}^{-\alpha} .
\end{aligned}
$$

Combining Eqs. (17) and (18), we obtain for $q=\frac{\rho S_{n}^{-\alpha}}{h-R_{-} / 2}$

$$
\begin{aligned}
\frac{\partial}{\partial t} q= & \alpha \rho S_{n}^{-(1+\alpha)} \dot{S}_{n}^{k l} \bar{\nabla}_{k} \bar{\nabla}_{l} q+\frac{2 \alpha \rho S_{n}^{-(1+\alpha)} \dot{S}_{n}^{k l}}{h-R_{-} / 2} \bar{\nabla}_{k} h \bar{\nabla}_{l} q \\
& -q^{2}\left(\alpha R_{-} H / 2-(1+n \alpha)\right)
\end{aligned}
$$

where $H=\sum_{i=1}^{n} \mathfrak{r}_{i}^{-1}=n S_{n-1} / S_{n} \geq n S_{n}^{-1 / n}$. By the maximum principle, this implies the following inequality for $Q=\sup _{S^{n}} q$ :

$$
\frac{d Q}{d t} \leq-Q^{2}\left(C(n, \alpha, \rho) R_{-} R_{+}^{\frac{1}{n \alpha}} Q^{\frac{1}{n \alpha}}-(1+n \alpha)\right)
$$

and we deduce

$$
Q \leq \max \left\{\frac{C(\alpha)}{R_{-}^{n \alpha} R_{+}}, C^{\prime}(\alpha) R_{-}^{-\frac{n \alpha}{1+n \alpha}} R_{+}^{-\frac{1}{1+n \alpha}} t^{-\frac{n \alpha}{1+n \alpha}}\right\} .
$$

From the definition of $Q$ and the estimate $h \leq 2 R_{+}$, we have

$$
\rho S_{n}^{-\alpha} \leq \max \left\{C(n, \alpha, \rho) R_{-}^{-n \alpha}, C^{\prime}(n, \alpha, \rho)\left(\frac{R_{+}}{R_{-}}\right)^{\frac{n \alpha}{1+n \alpha}} t^{-\frac{n \alpha}{1+n \alpha}}\right\} .
$$

We now proceed to obtain lower bounds on the speed and displacement. It is in this estimate that we require $\alpha \leq 1 / n$. The argument combines barrier arguments with a Harnack inequality (proved for isotropic Gauss curvature flows by Chow $[\mathbf{C h}]$ and for more general flows by the author [A7]). 
The Harnack estimate can be stated as follows for any solution of Equation (13) (see [A7], Theorem 5.6):

Theorem 7. For any smooth, strictly convex solution of (13) on $S^{n} \times[0, T)$,

$$
\frac{d}{d t}\left(\rho S_{n}^{-\alpha} t^{\frac{n \alpha}{n \alpha+1}}\right) \geq 0
$$

everywhere on $S^{n} \times(0, T)$.

Our lower speed estimate is the following:

Theorem 8. For $\alpha<1 / n$ the following holds for any smooth, strictly convex solution of Eq. (13):

$$
h(z, t) \leq h(z, 0)-C(\rho, n, \alpha) r_{+}\left(M_{0}\right)^{-\frac{2 n \alpha}{1-n \alpha}} t^{\frac{1}{1-n \alpha}}
$$

and

$$
\rho(z) S_{n}(z, t)^{-\alpha} \geq C^{\prime}(\rho, n, \alpha) r_{+}\left(M_{0}\right)^{-\frac{2 n \alpha}{1-n \alpha}} t^{\frac{n \alpha}{1-n \alpha}}
$$

for $0<t<C^{\prime \prime}(n, \rho, \alpha) r_{+}\left(M_{0}\right)^{1+n \alpha}$. For $\alpha=1 / n$ we have instead the estimates for each $\gamma>0$

$$
h(z, t) \leq h(z, 0)-C(n, \rho, \gamma) r_{+}\left(M_{0}\right)^{1+2 \gamma} t^{-\gamma} e^{-C^{\prime}(\rho, n, \gamma) r_{+}\left(M_{0}\right)^{2 n} t^{-n}}
$$

and

$$
\rho(z) S_{n}(z, t)^{-1 / n} \geq \bar{C}(n, \rho, \gamma) r_{+}\left(M_{0}\right)^{2 \gamma-1} t^{-\gamma} e^{-\bar{C}^{\prime}(\rho, n, \gamma) r_{+}\left(M_{0}\right)^{2 n} t^{-n}}
$$

for $0<t<\bar{C}^{\prime \prime}(n, \rho, \gamma) r_{+}\left(M_{0}\right)^{2}$.

Proof. For $n=1$ these estimates are proved in [A2], Theorem II2.4. Suppose $n \geq 2$.

In the case $\alpha<1 / n$ it suffices to use large spheres as barriers: Fix $z \in S^{n}$. Then $M_{0}$ is enclosed in a hemispherical region obtained by intersecting the sphere of radius $2 r_{+}\left(M_{0}\right)$ centred at $\bar{x}(z)$ with the half-space $\left\{y \in \mathbb{R}^{n+1}:\langle y, z\rangle \leq h(z)\right\}$. For any $\varepsilon<2 r_{+}(M)$ this hemispherical region is enclosed by the sphere $S_{\varepsilon}$ of radius $\left(\varepsilon^{2}+4 r_{+}(M)^{2}\right) /(2 \varepsilon)$ centred at the point $\bar{x}(z)-\left(4 r_{+}(M)^{2}-\varepsilon^{2}\right) /(2 \varepsilon) z$ (this sphere is chosen to have support function in direction $z$ equal to $h(z)+\varepsilon)$. We consider the evolution of these spheres for suitably small $\varepsilon$ : Since $\rho \geq \inf _{S^{n}} \rho$, a sphere of radius $r$ evolves in time $t$ to be contained inside a sphere of radius $\left(r^{1+n \alpha}-(1+n \alpha) \inf \rho t\right)^{1 /(1+n \alpha)}$ about the same centre, which is enclosed by the sphere of radius $r-\inf \rho r^{-n \alpha} t$. In particular, this applies for each of the sphere $S_{\varepsilon}$, and by the comparison principle $M_{t}$ is also enclosed by this smaller sphere. This implies the inequality

$$
\begin{aligned}
h(z, t)-h(z, 0) & \leq \varepsilon-(\inf \rho)\left(\frac{\varepsilon^{2}+4 r_{+}(M)^{2}}{2 \varepsilon}\right)^{-n \alpha} t \\
& \leq \varepsilon\left(1-\inf \rho\left(4 r_{+}(M)^{2}\right)^{-n \alpha} \varepsilon^{n \alpha-1} t\right) .
\end{aligned}
$$


In particular, choosing

$$
\varepsilon=\left(\frac{t \inf \rho}{2^{1+2 n \alpha} r_{+}\left(M_{0}\right)^{2 n \alpha}}\right)^{1 /(1-n \alpha)}
$$

we obtain

$$
h(z, t)-h(z, 0) \leq-\left(\frac{\inf \rho}{2^{1+2 n \alpha} r_{+}\left(M_{0}\right)^{2 n \alpha}}\right)^{1 /(1-n \alpha)} t^{1 /(1-n \alpha)}
$$

for $t \leq C(\rho, n, \alpha) r_{+}\left(M_{0}\right)^{1+n \alpha}$.

This estimate on the change in the support function can be converted to an estimate on the speed using the Harnack estimate from Theorem 7: Applying the estimate on the time interval $[t / 2, t]$, we have

$$
\begin{aligned}
C r_{+}\left(M_{0}\right)^{-\frac{2 n \alpha}{1+n \alpha}} t^{\frac{1}{1-n \alpha}} & \leq h(z, t / 2)-h(z, t) \\
& =\rho(z) \int_{t / 2}^{t} S_{n}(z, \tau)^{-\alpha} d \tau \\
& \leq t / 2 \sup _{\tau \in[t / 2, t]}\left(\rho S_{n}^{-\alpha}\right) .
\end{aligned}
$$

Theorem 7 then gives

$$
t^{\frac{n \alpha}{n \alpha+1}} \rho(z) S_{n}(z, t)^{-\alpha} \geq(t / 2)^{\frac{n \alpha}{n \alpha+1}} \sup _{[t / 2, t]} \rho S_{n}^{-\alpha}
$$

and hence

$$
\rho(z) S_{n}(z, t)^{-\alpha} \geq C^{\prime} r_{+}\left(M_{0}\right)^{-\frac{2 n \alpha}{1+n \alpha}} t^{1 /(1-n \alpha)-1} .
$$

In the case $1 / n$ the sphere barriers are not sufficient, and we instead work with graphical barriers. The displacement bound is a consequence of the following:

Lemma 9. Suppose $\alpha=1 / n$. If $M_{0}$ has bounded isoperimetric ratio, and lies in the region $x_{n+1} \geq 0$ and within the ball $\|x\| \leq R$, then $M_{t}$ lies inside the region

$$
x_{n+1} \geq C_{1} R^{1+2 \gamma} t^{-\gamma}\left(e^{-C_{2} R^{2 n-2}(r-2 R)^{2} t^{-n}}+e^{-C_{2} R^{2 n-2}(r+2 R)^{2} t^{-n}}\right)
$$

for $0 \leq t \leq C_{3} R^{2}$, where $r^{2}=\sum_{i=1}^{n} x_{i}^{2}$, and $C_{1}, C_{2}$ and $C_{3}$ are constants depending only on $n, \rho$, and $\gamma$.

Proof. We will show that the boundary of the region described is a graphical subsolution of the evolution Equation (14). In the special case of a radially symmetric function, we have

$$
K=\frac{u^{\prime \prime}\left(u^{\prime}\right)^{n-1}}{r^{n-1}\left(1+\left(u^{\prime}\right)^{2}\right)^{(n+2) / 2}},
$$


and so

$$
\dot{u}-\tilde{\rho}(D u) K^{1 / n} \sqrt{1+|D u|^{2}} \leq \dot{u}-\inf \rho\left(u^{\prime \prime}\right)^{1 / n}\left(\frac{u^{\prime}}{r}\right)^{1-1 / n}\left(1+\left(u^{\prime}\right)^{2}\right)^{-1 / n} .
$$

A direct computation shows that the function

$$
u(r, t)=C_{1} R^{1-2 \gamma} t^{\gamma}\left(e^{-C_{2} R^{2 n-2}(r-2 R)^{2} t^{-n}}+e^{-C_{2} R^{2 n-2}(r+2 R)^{2} t^{-n}}\right)
$$

makes the right-hand side of (19) non-positive on the region $r<R, t \leq$ $C_{3} R^{2}$, for any $\gamma \geq 0$, where $C_{1}, C_{2}$, and $C_{3}$ depend on $n, \rho$ and $\gamma$. Since the boundary of this region cannot intersect the hypersurface $M_{t}$, the comparison principle applies.

This gives the bounds in the theorem, since we can rotate and translate the solution to bring the initial supporting hyperplane to the hyperplane $x_{n+1}=0$, with $M_{0}$ satisfying the conditions of Lemma 9 with $R=2 r_{+}\left(M_{0}\right)$. Thus $h(z, 0)=0$. For positive sufficiently small $t$, Lemma 9 gives

$$
h(z, t)=-\inf _{M_{t}} x_{n+1} \leq-u(0, t)
$$

as required.

Similar barriers can also be constructed for each $\alpha<1 / n$.

The speed bound follows using Theorem 7 as for the previous cases.

We remark that the estimate for $\alpha<1 / n$ does not rely at all on the particular structure of the Gauss curvature flows - the same result holds for any strictly parabolic flow with speed homogeneous of degree less than 1 in the curvatures.

\section{Curvature control.}

In this section we prove that the ratio of the maximum and minimum principal curvatures remains bounded throughout the evolution, given the upper and lower speed bounds of the previous section. Our argument is an application of the parabolic maximum principle to the evolution equation for the curvature.

In the case $n=1$ the speed bounds above and below already give complete control on the curvatures. For the rest of this section we assume $n \geq 2$.

Theorem 10. Suppose $h: S^{n} \times[0, T]$ is a solution of Eq. (13) for which the isoperimetric ratio is bounded and the speed is bounded above and below - that is, there exist constants $C_{1}, C_{2}$ such that

$$
0<C_{1} \leq S_{n}(z, t) \leq C_{2}
$$

for every $z \in S^{n}$ and $t \in[0, T]$. Then there exist positive constants $C_{3}$ and $C_{4}$ depending only on $C_{1}, C_{2}, \rho, n$ and $\alpha$ such that

$$
\lambda_{i}(z, t) \geq \min \left\{C_{3} t^{n-1}, C_{4}\right\}
$$


for all $i \in\{1, \ldots, n\}, z \in S^{n}$, and $t \in[0, T]$.

Proof. We begin by computing the evolution equation for the matrix $\mathfrak{r}_{i j}=$ $\bar{\nabla}_{i} \bar{\nabla}_{j} h+\bar{g}_{i j} h$ under Eq. (13):

$$
\begin{aligned}
\frac{\partial}{\partial t} \mathfrak{r}_{i j}= & -\bar{\nabla}_{i} \bar{\nabla}_{j}\left(\rho S_{n}^{-\alpha}\right)-\bar{g}_{i j} \rho S_{n}^{-\alpha} \\
= & \alpha \rho S_{n}^{-(1+\alpha)} \dot{S}_{n}^{k l} \bar{\nabla}_{i} \bar{\nabla}_{j} \mathfrak{r}_{k l}-\alpha(1+\alpha) \rho S_{n}^{-(2+\alpha)} \bar{\nabla}_{i} S_{n} \bar{\nabla}_{j} S_{n} \\
& +\alpha \rho S_{n}^{-(2+\alpha)} \ddot{S}_{n}^{k l m n} \bar{\nabla}_{i} \mathfrak{r}_{k l} \bar{\nabla}_{j} \mathfrak{r}_{m n}-\bar{g}_{i j} \rho S_{n}^{-\alpha}-S_{n}^{-\alpha} \bar{\nabla}_{i} \bar{\nabla}_{i} \rho \\
& +\alpha S_{n}^{-(1+\alpha)} \bar{\nabla}_{i} \rho \bar{\nabla}_{j} S_{n}+\alpha S_{n}^{-(1+\alpha)} \bar{\nabla}_{j} \rho \bar{\nabla}_{i} S_{n} .
\end{aligned}
$$

In the first term here we apply the identity (12), to yield:

$$
\begin{aligned}
\frac{\partial}{\partial t} \mathfrak{r}_{i j}= & \alpha \rho S_{n}^{-(1+\alpha)} \dot{S}_{n}^{k l} \bar{\nabla}_{k} \bar{\nabla}_{l} \mathfrak{r}_{i j}-\alpha(1+\alpha) \rho S_{n}^{-(2+\alpha)} \bar{\nabla}_{i} S_{n} \bar{\nabla}_{j} S_{n} \\
& +\alpha \rho S_{n}^{-(2+\alpha)} \ddot{S}_{n}^{k l m n} \bar{\nabla}_{i} \mathfrak{r}_{k l} \bar{\nabla}_{j} \mathfrak{r}_{m n} \\
& +(n \alpha-1) \rho S_{n}^{-\alpha} \bar{g}_{i j}-\alpha \rho S_{n}^{-(1+\alpha)} \dot{S}_{n}^{k l} \bar{g}_{k l} \mathfrak{r}_{i j} \\
& +S_{n}^{-\alpha} \bar{\nabla}_{i} \bar{\nabla}_{j} \rho+\alpha S_{n}^{-(1+\alpha)} \bar{\nabla}_{i} \rho \bar{\nabla}_{j} S_{n}+\alpha S_{n}^{-(1+\alpha)} \bar{\nabla}_{j} \rho \bar{\nabla}_{i} S_{n} .
\end{aligned}
$$

We wish to obtain an upper bound for the eigenvalues of $\mathfrak{r}_{i j}$, so the second term on the first line and the last term on the second line are good terms since they are negative. The first term of the first line is an elliptic operator, and so is non-positive at a point and direction where a maximum eigenvalue occurs. The first term of the second line we estimate using the concavity of the $n$th root of the determinant as a function of the components of the matrix, which is equivalent to the inequality

$$
\left(\ddot{S}_{n}^{k l m n}-\frac{n-1}{n S_{n}} \dot{S}_{n}^{k l} \dot{S}_{n}^{m n}\right) \xi_{k l} \xi_{m n} \leq 0
$$

for any symmetric matrix $\xi$. Finally, the first term on the last line is bounded, and the other two terms of the last line can be estimated in terms of the good second term of the first line:

$$
\left|\bar{\nabla}_{i} \rho \bar{\nabla}_{i} S_{n}\right| \leq C \varepsilon\left|\bar{\nabla}_{i} S_{n}\right|^{2}+C \varepsilon^{-1}
$$

for any $\varepsilon>0$. Combining these estimates, we obtain

$$
\frac{\partial}{\partial t} \mathfrak{r}_{i j} \leq \alpha \rho S_{n}^{-(1+\alpha)} \dot{S}_{n}^{k l} \bar{\nabla}_{k} \bar{\nabla}_{l} \mathfrak{r}_{i j}+C S_{n}^{-\alpha} \bar{g}_{i j}-\alpha \rho S_{n}^{-(1+\alpha)} \dot{S}_{n}^{k l} \bar{g}_{k l} \mathfrak{r}_{i j} .
$$

The last term here will allow us to obtain an estimate independent of initial data: We have $S_{n}^{k l} \bar{g}_{k l}=S_{n-1} / S_{n}$, and the Newton inequalities [Mi] give

$$
S_{n-1} \geq S_{n}^{\frac{n-2}{n-1}} S_{1}^{\frac{1}{n-1}} \geq C S_{n}^{\frac{n-2}{n-1}} \mathfrak{r}_{\max }^{\frac{1}{n-1}}
$$


Now work at a point and time where a maximum eigenvalue is attained, and suppose $i=j$ and $e_{i}$ is the eigenvector of $\mathfrak{r}$ with the largest eigenvalue. Then the first term in the evolution equation is negative, and

$$
\frac{\partial \mathfrak{r}_{\max }}{\partial t} \leq C S_{n}^{-\alpha} \bar{g}_{i j}-C S_{n}^{-\left(\frac{n}{n-1}+\alpha\right)} \mathfrak{r}_{\max }^{\frac{n}{n-1}} \leq C S_{n}^{-\alpha}\left(\bar{g}_{i j}-C S_{n}^{-\frac{n}{n-1}} \mathfrak{r}_{\max }^{\frac{n}{n-1}}\right) .
$$

Given the bound below on $S_{n}^{-\alpha}$, the bracket is negative provided $\mathfrak{r}_{\text {max }}$ is sufficiently large. For the same reason the coefficient in front of the bracket does not become small, and we have for $\mathfrak{r}_{\max }$ sufficiently large

$$
\frac{d}{d t} \mathfrak{r}_{\max } \leq-C \mathfrak{r}_{\max }^{n /(n-1)}
$$

The result now follows by the parabolic maximum principle and comparison with the solution of the ordinary differential equation $d u / d t=-C u^{n /(n-1)}$.

Next we observe that this automatically provides an upper bound on the principal curvatures:

Proposition 11. Let $\mathcal{W}$ be a positive definite symmetric matrix for which $\mathcal{W} \geq \varepsilon \operatorname{Id}$ and $\operatorname{det} \mathcal{W} \leq C$. Then $\mathcal{W} \leq C \varepsilon^{-(n-1)} \mathrm{Id}$.

Proof. Number the eigenvalues $\lambda_{1}$ of $\mathcal{W}$ in ascending order: $\lambda_{1} \leq \lambda_{2} \leq \cdots \leq$ $\lambda_{n}$. Then

$$
\lambda_{n}=\frac{K}{\lambda_{1} \lambda_{2} \ldots \lambda_{n-1}} \leq \frac{K}{\lambda_{1}^{n-1}},
$$

where $K=\lambda_{1} \ldots \lambda_{n}=\operatorname{det} \mathcal{W}$.

In particular, the upper speed bound of Theorem 6 and the lower curvature bound of Theorem 10 imply an upper curvature bound:

Corollary 12. Under the conditions of Theorem 10 there exist constants $C_{5}$ and $C_{6}$ such that

$$
\mathcal{W} \leq \max \left\{C_{5} t^{-(n-1)^{2}}, C_{6}\right\} \mathrm{Id}
$$

\section{Convergence to a point.}

In this section we prove that any solution of Eq. (6) with a smooth, strictly convex initial hypersurface converges to a point in finite time. In the special case of isotropic flows $(\rho \equiv 1)$ this was proved by K.S. Chou [Ts] for $\alpha=1$ and by Ben Chow $[\mathbf{C h} \mathbf{1}]$ for other $\alpha$. While we only need the result for $\alpha \leq 1 / n$, we give a proof which works for larger $\alpha$ as well.

Theorem 13. For any $\alpha>0$ and positive $\rho \in C^{\infty}\left(S^{n}\right)$, and any smooth, strictly convex hypersurface $M_{0} \subset \mathbb{R}^{n+1}$, the hypersurfaces $M_{t}$ given by the solution of Eq. (6) exist for a finite time $T$ and converge in Hausdorff distance to $p \in \mathbb{R}^{n+1}$ as $t$ approaches $T$. 
Proof. The maximal time of existence must be finite: By the comparison principle, if $M_{0}$ is enclosed by a sphere $S_{r(0)}^{n}(q)$ for some $r>0$ and $q \in \mathbb{R}^{n+1}$, then for all $t$ in the interval of existence, $M_{t}$ is enclosed by the sphere $S_{r(t)}^{n}(q)$, where

$$
r(t)=\left(r(0)^{1+n \alpha}-\left(\inf _{S^{n}} \rho\right)(1+n \alpha) t\right)^{1 /(1+n \alpha)} .
$$

$r(t)$ converges to zero in finite time, and $M_{t}$ cannot exist beyond this time.

Consider again the estimate (22) for the evolution of the curvature. We also have the evolution equation

$$
\begin{aligned}
\frac{\partial}{\partial t} h & =-\rho S_{n}^{-\alpha} \\
& =\alpha \rho S_{n}^{-(1+\alpha)} \dot{S}_{n}^{k l} \bar{\nabla}_{k} \bar{\nabla}_{l} h+\alpha \rho S_{n}^{-(1+\alpha)} \dot{S}_{n}^{k l} \bar{g}_{k l} h-(1+n \alpha) \rho S_{n}^{-\alpha} .
\end{aligned}
$$

Combining these, we obtain

$$
\begin{aligned}
\frac{\partial}{\partial t}\left(\mathfrak{r}_{i j}+A h \bar{g}_{i j}\right) \leq & \alpha \rho S_{n}^{-(1+\alpha)} \dot{S}_{n}^{k l} \bar{\nabla}_{k} \bar{\nabla}_{l}\left(\mathfrak{r}_{i j}+A h \bar{g}_{i j}\right) \\
& +(C-A(1+n \alpha)) S_{n}^{-\alpha} \bar{g}_{i j} \\
& +\left(A h-\mathfrak{r}_{i j}\right) \alpha \rho S_{n}^{-(1+\alpha)} \dot{S}_{n}^{k l} \bar{g}_{k l} .
\end{aligned}
$$

Choose $A=C /(1+n \alpha)$, so that the last term of the first line vanishes. Also note that since the hypersurfaces are contracting, we have $h \leq h_{0}=$ $\sup _{S^{n}} h(z, 0)$ as long as the solution exists. Therefore we have, writing $q_{i j}=$ $\mathfrak{r}_{i j}+A h \bar{g}_{i j}$,

$$
\frac{\partial}{\partial t} q_{i j} \leq \alpha \rho S_{n}^{-(1+\alpha)} \dot{S}_{n}^{k l} \bar{\nabla}_{k} \bar{\nabla}_{l} q_{i j}-\alpha \rho S_{n}^{-(1+\alpha)} \dot{S}_{n}^{k l} \bar{g}_{k l}\left(q_{i j}-2 h_{0} \bar{g}_{i j}\right)
$$

and hence by the parabolic maximum, the maximum eigenvalue of $q_{i j}$ is decreasing if it is larger than $2 h_{0}$. Since the initial hypersurface is smooth and strictly convex, $q_{i j}$ is bounded at $t=0$. Therefore we have a uniform bound on $q_{i j}$ and hence $\mathfrak{r}_{i j}$ throughout the interval of existence.

Suppose the inradius of the hypersurfaces $M_{t}$ do not converge to zero that is, the solution exists for a maximal time interval $[0, T)$, but there is some ball of positive radius that remains enclosed by the solution throughout. By the argument in Section 5, the speed remains bounded throughout the interval of existence.

By Proposition 11 this also implies a bound on the curvature, so that the hypersurfaces remain uniformly smooth and strictly convex on the time interval $[0, T)$. It follows that there exists a subsequence of times $\left\{t_{k}\right\}$ converging to $T$ such that $M_{t_{k}}$ converges in $C^{\infty}$ to a smooth, strictly convex limit $M_{T}$. Furthermore, the $C^{\infty}$ convergence implies that all time derivatives converge, so that in fact $M_{t}$ approaches $M_{T}$ in $C^{\infty}$ as $t$ approaches 
$T$. Hence we have a smooth solution on $[0, T]$, and the short-time existence result implies that this can be extended beyond $T$, contradicting the assumption that $T$ was maximal.

Therefore the inradius converges to zero. Since $\mathfrak{r}_{i j}$ is uniformly bounded, this also implies that the circumradius converges to zero, and the hypersurfaces converge to a point.

Theorem 13 implies a bound below on the time of existence of solutions with smooth and strictly convex initial data, in terms of the inradius of the initial hypersurface, $\rho$, and $\alpha$ : Any sphere which is initially enclosed by the hypersurface acts as a barrier, preventing the hypersurface from contracting to a point too quickly.

\section{Short-time existence.}

In this section we prove the following:

Theorem 14. For any positive $\rho \in C^{\infty}\left(S^{n}\right), \alpha \leq 1 / n$, and open bounded convex region $\Omega_{0} \subset \mathbb{R}^{n+1}$, there exists a smooth, strictly convex solution $x_{t}: S^{n} \times(0, T)$ of Eq. (6) which converges to $M_{0}=\partial \Omega_{0}$ in Hausdorff distance as $t$ approaches zero. Any other such solution $y_{t}: S^{n} \times\left(0, T^{\prime}\right)$ is given by $x_{t} \circ \varphi$ for some smooth diffeomorphism $\varphi$ of $S^{n}$.

At the end of the section we also prove the existence and uniqueness of viscosity solutions for arbitrary convex initial data and arbitrary $\alpha>0$.

8.1. Existence. In order to construct a solution which approaches $M_{0}$ at the initial time, we consider a family of smooth, strictly convex hypersurfaces $M_{0}^{(\varepsilon)}$ which approach $M_{0}$ in Hausdorff distance as $\varepsilon$ approaches zero. By Theorem 13, for each $\varepsilon>0$ there exists a unique solution $M_{t}^{(\varepsilon)}$ of Eq. (6) with initial condition $M_{0}^{(\varepsilon)}$, which converges to a point in finite time $T_{\varepsilon}>0$.

Then we have

$$
\begin{aligned}
d_{H}\left(M_{0}, M_{t}^{(\varepsilon)}\right) & \leq d_{H}\left(M_{0}, M_{0}^{(\varepsilon)}\right)+d_{H}\left(M_{0}^{(\varepsilon)}, M_{t}^{(\varepsilon)}\right) \\
& \leq d_{H}\left(M_{0}, M_{0}^{(\varepsilon)}\right)+C t^{1 /(1+n \alpha)} .
\end{aligned}
$$

By Theorem 10 and Corollary 12, the hypersurfaces $M_{t}^{(\varepsilon)}$ satisfy bounds above and below on the principal curvatures, uniformly in $\varepsilon$ over every compact subset of $S^{n} \times(0, T)$. It follows from the regularity theory for solutions of uniformly parabolic equations concave in the second derivatives $([\mathbf{K}]$, Theorem 5.5) that there are also bounds on all higher derivatives of the curvatures, uniformly in $\varepsilon$ over every compact subset of $S^{n} \times(0, T)$. It follows from the Arzela-Ascoli theorem that there exists a sequence $\varepsilon_{k}$ approaching zero such that $\left\{M_{t}^{\left(\varepsilon_{k}\right)}\right\}$ converges in $C^{\infty}$ to a family of hypersurfaces $\left\{M_{t}\right\}$ satisfying the same bounds. In particular, $\left\{M_{t}\right\}$ satisfies Eq. (6) on $S^{n} \times$ 
$(0, T), M_{t}$ is smooth and strictly convex for each $t>0$, and $d_{H}\left(M_{0}, M_{t}\right) \leq$ $C t^{1 /(1+n \alpha)}$.

8.2. Uniqueness. Suppose we have two solutions $\left\{M_{t}^{(1)}\right\}$ and $\left\{M_{t}^{(2)}\right\}$ of Eq. (6), both converging to $M_{0}$ in Hausdorff distance as $t \rightarrow 0$, and denote by $h_{t}^{(i)}$ the corresponding support functions. Fix $\varepsilon>0$. Then there exists $t_{0}(\varepsilon)>0$ such that $\left|h_{t}^{(i)}(z)-h_{0}(z)\right|<\varepsilon$ for $i=1,2$ and all $z \in S^{n}$ and $t \in\left(0, t_{0}(\varepsilon)\right)$. Choose a smooth, strictly convex hypersurface $M_{0}^{(\varepsilon)}$ with support function $h_{0}^{(\varepsilon)}$ such that $h_{0}(z)-2 \varepsilon<h_{0}^{(\varepsilon)}(z)<h_{0}(z)-\varepsilon$. Without loss of generality we assume that the origin is at the centre of a ball of radius $r_{-}\left(M_{0}\right)$ enclosed by $M_{0}$. Then $r_{-}\left(M_{0}\right) \leq h_{0}(z) \leq 2 r_{+}\left(M_{0}\right)$. It follows that

$$
h_{0}^{(\varepsilon)}(z)<h_{0}(z)-\varepsilon<h_{t}^{(i)}(z)<h_{0}(z)+\varepsilon<\left(1+\frac{3 \varepsilon}{r_{-}\left(M_{0}\right)-2 \varepsilon}\right) h_{0}^{(\varepsilon)}
$$

for $i=1,2$ and all $z \in S^{n}$ and $t \in\left(0, t_{0}(\varepsilon)\right)$. The comparison principle and the scaling property given by Eq. (15) then imply

$$
h_{\tau}^{(\varepsilon)}(z)<h_{t+\tau}^{(i)}(z)<(1+\lambda) h_{(1+\lambda)^{-(1+n \alpha)} \tau}^{(\varepsilon)}(z)
$$

for all $\tau \geq 0$ for which these all exist, where $\lambda=\frac{3 \varepsilon}{r_{-}\left(M_{0}\right)-2 \varepsilon}$. Consequently,

$$
\begin{aligned}
\left|h_{t+\tau}^{(2)}(z)-h_{t+\tau}^{(1)}(z)\right| & \leq(1+\lambda) h_{(1+\lambda)^{-(1+n \alpha)} \tau}^{(\varepsilon)}(z)-h_{\tau}^{(\varepsilon)}(z) \\
& \leq \lambda h_{(1+\lambda)^{-(1+n \alpha)_{\tau}}}^{(\varepsilon)}(z)+\left(h_{(1+\lambda)^{-(1+n \alpha)} \tau}^{(\varepsilon)}(z)-h_{\tau}^{(\varepsilon)}(z)\right) \\
& \leq 2 r_{+}\left(M_{0}\right) \lambda+C\left(\left|1-(1+\lambda)^{-(1+n \alpha)}\right| \tau\right)^{1 /(1+n \alpha)} \\
& \leq C \lambda+C(\lambda \tau)^{1 /(1+n \alpha)} .
\end{aligned}
$$

Here we used Theorem 6 to obtain the second-last line. Now take $t \rightarrow 0$. Since $\varepsilon>0$ is arbitrary and $C$ independent of $\varepsilon$, we have for each $\tau>0$ and $z \in S^{n}$

$$
h_{\tau}^{(2)}(z)=h_{\tau}^{(1)}(z)
$$

Note that the proof presented here does not rely strongly on the particular structure of the evolution Equation (6). In particular, the uniqueness argument is valid for any flow by a monotone, positively homogeneous function of curvature, since the bound on the change in the support function given in Theorem 6 also holds for all such evolution equations. The existence argument requires a speed bound and regularity estimates independent of initial data.

We now proceed to the case $\alpha>1 / n$ : In this case (as we show in Section 12) one cannot expect to produce smooth solutions from arbitrary convex initial hypersurfaces. Instead we will work with a weaker notion of solution: A family of convex regions $\left\{\Omega_{t}\right\}_{0<t<T}$ is called a viscosity solution of Eq. (6) 
if the following conditions hold: First, for any smooth, strictly convex hypersurface $M_{0}$ contained in $\Omega_{t_{0}}$ for some $t_{0} \in(0, T)$, the hypersurfaces $M_{t}$ given by solving (6) are contained in $\Omega_{t_{0}+t}$ for all $t \in\left[0, T-t_{0}\right)$ in the domain of existence of the $M_{t}$. Second, for any smooth, strictly convex hypersurface $M_{0}$ which encloses $\Omega_{t_{0}}$ for some $t_{0} \in(0, T)$, the hypersurfaces $M_{t}$ enclose $\Omega_{t_{0}+t}$ for all $t \in\left[0, T-t_{0}\right)$.

Theorem 15. For any smooth positive $\rho \in C^{\infty}\left(S^{n}\right), \alpha>0$, and any open bounded convex region $\Omega_{0} \subset \mathbb{R}^{n+1}$, there exists a unique viscosity solution $\left\{\Omega_{t}\right\}_{0<t<T}$ which converges to $\Omega_{0}$ is Hausdorff distance as $t$ approaches zero. $\Omega_{t}$ converges to a point as $t$ approaches $T$.

Proof. We use the same construction as presented in the proof of Theorem 14, producing a solution $\left\{M_{t}^{(\varepsilon)}\right\}$ for each $\varepsilon>0$, with $M_{0}^{(\varepsilon)}$ approaching $\partial \Omega_{0}$ in Hausdorff distance as $\varepsilon$ approaches zero. We specify further that $M_{0}^{(\varepsilon)}$ is contained in $\Omega_{0}$ for all $\varepsilon>0$, and is increasing in $\varepsilon$.

For $\varepsilon$ sufficiently small, we can choose an origin for $\mathbb{R}^{n+1}$ and radii $R>$ $r>0$ such that the ball $B_{r}(0)$ is enclosed by all of the hypersurfaces $M_{0}^{(\varepsilon)}$, and the ball $B_{R}(0)$ contains all of the hypersurfaces $M_{0}^{(\varepsilon)}$. By the comparison principle, there exists $\delta>0$ such that the ball $B_{r / 2}(0)$ is enclosed by all the hypersurfaces $M_{t}^{(\varepsilon)}$ for $t \in[0, \delta]$. The hypersurfaces also remain enclosed by the ball $B_{R}(0)$.

It follows that the support functions $h_{t}^{(\varepsilon)}(z)$ are uniformly Lipschitz: By the Formula (9), we have $|\bar{x}|^{2}=h^{2}+|\bar{\nabla} h|^{2}$, and re-arrangement gives $|\bar{\nabla} h|^{2} \leq|\bar{x}|^{2} \leq R^{2}$, which is a uniform Lipschitz bound.

Furthermore, the displacement bound and the speed bound of Theorem 6 show that $h_{t}^{(\varepsilon)}$ is Hölder continuous in $t$, uniformly in $\varepsilon$ and $z$, and also uniformly Lipschitz on compact subsets of $(0, \delta)$. Therefore $h^{(\varepsilon)}(z, t)$ is a Hölder continuous function on $S^{n} \times[0, \delta]$, uniformly in $\varepsilon$. By the ArzelaAscoli theorem, there exists a sequence $\varepsilon_{k}$ approaching zero which converges to a limit $h(z, t)$ satisfying the same estimates. By the Blaschke selection theorem, each of the functions $h_{t}=h(., t)$ is the support function of a convex region $\Omega_{t}$, and the same argument as in the proof of Theorem 14 shows that $\Omega_{t}$ approaches $\Omega_{0}$ in Hausdorff distance as $t$ approaches zero.

We need to prove that the family $\left\{\Omega_{t}\right\}$ is a viscosity solution. The first condition is easily checked: If $M_{0}^{\prime}$ is contained within $\Omega_{0}$, then $M_{0}^{\prime}$ is also enclosed by $M_{0}^{(\varepsilon)}$ for $\varepsilon>0$ sufficiently small. By the comparison principle, the resulting solution $M_{t}^{\prime}$ is enclosed by $M_{t}^{(\varepsilon)}$ for $t>0$, and also $M_{t}^{(\varepsilon)}$ is increasing in $\varepsilon$ and converges to $\partial \Omega_{t}$ as $\varepsilon$ approaches zero. Therefore $M_{t}^{\prime}$ is contained in $\Omega_{t}$ for $t>0$. 
The second condition also follows easily: Any hypersurface $M_{0}^{\prime}$ which encloses $\Omega_{0}$ also encloses all of the hypersurfaces $M_{0}^{(\varepsilon)}$, and so by the comparison principle $M_{t}^{\prime}$ encloses $M_{t}^{(\varepsilon)}$ for $\varepsilon>0$ and $t>0$, and so also encloses the limit $\partial \Omega_{t}$.

The uniqueness statement in Theorem 15 follows exactly as in the proof of uniqueness in Theorem 14, and the same argument shows that the regions $\Omega_{t}$ converge to a point.

\section{Application: The affine normal flow.}

In this section we apply the speed and curvature bounds of the previous sections to give a short proof of the following theorem:

Theorem 16. Let $\alpha=1 /(n+2)$ and $\rho \equiv 1$. For any convex open region $\Omega_{0} \subset \mathbb{R}^{n+1}$ there exists a smooth family of strictly convex embeddings $x_{t}$ : $S^{n} \rightarrow \mathbb{R}^{n+1}$ satisfying Eq. (6) for which the Hausdorff distance between $M_{t}=x_{t}\left(S^{n}\right)$ and $M_{0}$ approaches zero as $t \rightarrow 0$. Any other such solution $\left\{\tilde{x}_{t}\right\}$ is related to $\left\{x_{t}\right\}$ by composition with a time-independent diffeomorphism. $M_{t}$ converges to a point $p \in \mathbb{R}^{n+1}$ as $t$ approaches a finite time $T$, and

$$
\tilde{M}_{t}=\left(\frac{\operatorname{Vol}\left(S^{n}\right)}{\operatorname{Vol}\left(M_{t}\right)}\right)^{1 /(n+1)}\left(M_{t}-p\right)
$$

converges in $C^{\infty}$ to an ellipsoid centred at the origin.

This theorem was proved in the case of smooth, strictly convex $M_{0}$ in [A4]. The results of Section 6 allow us to give a proof which works also for singular initial hypersurfaces. The argument is also considerably simpler because it avoids the complicated third-derivative estimate which was the key to the proof in [A4]. On the other hand, we use the result that elliptic affine hyperspheres are ellipsoids, which was not necessary for the proof in [A4].

Proof. By Section 8, we have a unique solution of Eq. (6) with the given initial condition. Since this is smooth and strictly convex for $t>0$, the result of Theorem 13 implies that this solution converges to $p \in \mathbb{R}^{n+1}$ in finite time $T$.

In the proof we use the fact that the evolution equation is invariant under the action of the special affine group: If $\left\{M_{t}\right\}$ is a family of hypersurfaces moving under Eq. (6), then $\left\{L\left(M_{t}\right)\right\}$ is also such a family, for any special affine transformation $L$.

Fix $t \in[0, T)$. There exists a special affine transformation $L_{t}$ such that $L_{t}\left(M_{t}\right)$ has

$$
C_{-} \operatorname{Vol}\left(M_{t}\right)^{1 /(n+1)} \leq \operatorname{Vol}\left(S^{n}\right)^{1 /(n+1)} h_{L_{t}\left(M_{t}\right)}(z) \leq C_{+} \operatorname{Vol}\left(M_{t}\right)^{1 /(n+1)},
$$

for some absolute constants $C_{ \pm}$. 
We consider the solution $\tilde{h}$ given by the scaling relation (15):

$$
\tilde{h}(z, \tau)=\left(\frac{\operatorname{Vol}\left(S^{n}\right)}{\operatorname{Vol}\left(M_{t}\right)}\right)^{1 /(n+1)} h_{L_{t}}\left(z, t+\left(\frac{\operatorname{Vol}\left(S^{n}\right)}{\operatorname{Vol}\left(M_{t}\right)}\right)^{-2 /(n+2)} \tau\right),
$$

where $h_{L}$ is the support function of the convex body obtained by applying the special affine transformation $L$ to the body with support function $h$. Then $C_{-} \leq \tilde{h}(z, 0) \leq C_{+}$. By the comparison principle we also have $\frac{1}{2} C_{-} \leq \tilde{h}(z, \tau) \leq C_{+}$for $\tau \in[0, \delta]$, where $\delta=\left(C_{-}\right)^{2(n+1) /(n+2)}(1-$ $\left.2^{-2(n+1) /(n+2)}\right)(n+2) / 2(n+1)$. Hence on the interval $[\delta / 2, \delta]$ there are uniform speed and displacement bounds (by Theorem 6), a uniform lower bound on the speed (by Theorem 8), uniform bounds above and below on the principal curvatures (by Theorem 10 and Corollary 12), and uniform bounds on all higher derivatives of the curvature (by Theorem 5.5 of $[\mathbf{K}]$ ).

It follows that the original solution satisfies uniform bounds on all quantities which are both scaling invariant and special-affine invariant, on the time interval $\left[t+\frac{1}{2} C \operatorname{Vol}\left(M_{t}\right)^{2 /(n+2)}, t+C \operatorname{Vol}\left(M_{t}\right)^{2 /(n+2)}\right]$, for some absolute constant $C$. Since $t$ is arbitrary, we have such bounds on the entire interval $[T / 2, T)$.

Therefore there exists a sequence $\left\{t_{k}\right\}$ approaching $T$, and a sequence of special affine transformations $\left\{L_{k}\right\}$, such that the hypersurfaces $\left\{L_{k}\left(\tilde{M}_{t_{k}}\right)\right\}$ converge in $C^{\infty}$ to a smooth, strictly convex limit $\tilde{M}_{T}$.

Suppose $\tilde{M}_{T}$ does not satisfy the condition $K^{1 /(n+2)}=c\langle x, \nu\rangle$ for some $c>0$ and some choice of origin. Then the time derivative of $\mathcal{Z}_{\natural, 1 /(n+2)}$ on $\tilde{M}_{T}$ is strictly negative, by Theorem 3 . By the $C^{\infty}$ convergence and scaling, there exists $k_{0}, \delta>0$, and $\varepsilon>0$ such that whenever $k \geq k_{0}$ we have

$$
\mathcal{Z}_{\natural, 1 /(n+2)}\left(M_{t_{k}+\delta \operatorname{Vol}\left(M_{t_{k}}\right)^{2 /(n+2)}}\right) \leq \mathcal{Z}_{\natural, 1 /(n+2)}\left(M_{t_{k}}\right)-\varepsilon .
$$

But since $\mathcal{Z}_{\natural, 1 /(n+2)}$ is non-increasing, this would imply $\mathcal{Z}_{\natural, 1 /(n+2)}\left(\tilde{M}_{t_{k}}\right) \rightarrow$ $-\infty$ as $k \rightarrow \infty$, which is impossible. Therefore $\tilde{M}_{T}$ satisfies the required condition.

By Theorem 1 of $[\mathbf{C a}]$, a smooth, strictly convex hypersurface satisfies the condition $K^{1 /(n+2)}=c\langle x, \nu\rangle$ if and only if it is an ellipsoid.

The stronger convergence statements in the Theorem follow by considering the linearization of the evolution Equation (13) about the space of ellipsoids - a direct calculation shows that this space is strictly linearly stable, so Proposition 9.2.3 of [Lu] and a scaling argument implies that $M_{t}$ converges in $C^{\infty}$ to the ellipsoid $\tilde{M}_{T}$ after rescaling. The details of this argument are given for a related evolution equation in [A10], Propositions 40-41. 
Corollary 17. For any open bounded convex region $\Omega_{0} \subset \mathbb{R}^{n+1}$, the following generalised affine isoperimetric inequality holds:

$$
\lim _{t \rightarrow 0} \mathcal{Z}_{\natural, \frac{1}{n+2}}\left(\Omega_{t}\right) \geq 1
$$

with equality if and only if $\Omega_{0}$ is an ellipse.

In effect this result allows a definition of the affine surface area for convex hypersurfaces which may be non-smooth or non-strictly convex, in such a way that the affine isoperimetric inequality remains true. A related extension of the affine surface area has been given in $[\mathbf{L e}]$.

\section{Proof of the main Theorems.}

In this section we complete the proofs of Theorems 1 and 2. The proof is similar to that presented in the special case of the affine normal flow in the previous section, but is somewhat simpler because we do not require the machinery of affine corrections to obtain bounded isoperimetric ratios.

Section 8 provides us with a unique solution $\left\{M_{t}\right\}$ of Eq. (6) for a short time, and Theorem 13 ensures that this solution remains smooth until it converges to a point $p \in \mathbb{R}^{n+1}$ in finite time $T$. In the case $\alpha>1 /(n+$ 2 ), Theorem 4 gives a uniform bound on the isoperimetric ratios of the hypersurfaces $M_{t}$ throughout the interval $[0, T]$. In the case described in Theorem 2, we also have such an estimate by hypothesis.

Theorems 6, 8 and 10 and Corollary 12 therefore imply uniform bounds above and below on the principal curvatures of the rescaled hypersurfaces $\left\{\tilde{M}_{t}\right\}$ defined by rescaling to fixed enclosed volume about the point $p$. Theorem 5.5 of $[\mathbf{K}]$ and Schauder estimates ([Li] , Theorem 4.9) imply uniform bounds on all higher derivatives of curvature. It follows that there exists a subsequence of times $\left\{t_{k}\right\}$ approaching $T$ for which the hypersurfaces $\tilde{M}_{t_{k}}$ converge in $C^{\infty}$ to a smooth, strictly convex limit $\tilde{M}_{T}$. By the same argument as that in Section $9, \tilde{M}_{t}$ satisfies the equation $\langle x, \nu\rangle=c \rho K^{\alpha}$ for some $c>0$. The convergence in $C^{\infty}$ of $\tilde{M}_{t}$ to $\tilde{M}_{T}$ as $t$ approaches $T$ follows from Theorem 2 of $[\mathbf{A} 6]$.

\section{Application: Non-trivial homothetic solutions.}

In this section we give an application of Theorem 2 to prove the existence of homothetically contracting solutions of the isotropic flows

$$
\frac{\partial}{\partial t} x=-K^{\alpha} \nu
$$

for sufficiently small $\alpha$. The idea is to consider the evolution of hypersurfaces close to the sphere $S^{n}$, possessing suitable symmetries. Precisely, our result is the following: 
Theorem 18. Let $\Gamma$ be a proper subgroup of $S O(n+1)$ such that for every $z \in S^{n}$, the orbit of $z$ under $\Gamma$ spans $\mathbb{R}^{n+1}$ (that is, the inclusion of $\Gamma$ in $S O(n+1)$ is an irreducible representation). Let $\lambda$ be the smallest eigenvalue corresponding to a non-trivial $\Gamma$-invariant spherical harmonic $\varphi$. Then for $\alpha \in(0,1 /(\lambda-n))$ there exists a non-spherical, $\Gamma$-symmetric, smooth, strictly convex hypersurface satisfying the identity $\langle x, \nu\rangle=K^{\alpha}$.

Proof. The linearization of the normalised isotropic Equation (6) about the sphere solution $h \equiv=1$ is given by

$$
\frac{\partial}{\partial t} \eta=\alpha(\Delta+n) u+u
$$

where $\Delta$ is the Laplacian on $S^{n}$. In particular, for $\alpha \in(0,1 /(\lambda-n))$ the $h \equiv 1$ solution is strictly unstable in the direction $\varphi$. By $[\mathbf{L u}]$, Theorem 9.1.3, there exists a $\Gamma$-symmetric solution $\left\{M_{t}\right\}$ of Eq. (6) which converges to $h \equiv 1$ as $t \rightarrow-\infty$ and diverges exponentially from $h \equiv 1$.

We observe that $\Gamma$-symmetry of a convex hypersurface implies a bound on the isoperimetric ratio:

Lemma 19. For any $\Gamma$ satisfying the conditions of Theorem 18, there exists a constant $C$ such that every $\Gamma$-symmetric convex hypersurface $M \subset \mathbb{R}^{n+1}$ satisfies $r_{+}(M) / r_{-}(M) \leq C$.

Proof. If this is not the case, then we can find a sequence of $\Gamma$-symmetric convex hypersurfaces $M_{k}$ such that $r_{+}\left(M_{k}\right)=1$ and $r_{-}\left(M_{k}\right) \leq 1 / k$. By the Blaschke selection theorem ([Sc], Theorem 2.5.14) we can choose a subsequence $M_{k^{\prime}}$ which converges in Hausdorff distance to a limit $M_{\infty}$ which is again $\Gamma$-symmetric but has $r_{-}\left(M_{\infty}\right)=0$ and $r_{+}\left(M_{\infty}\right)=1$. It follows that $M_{\infty}$ is contained in a lower-dimensional subspace of $\mathbb{R}^{n+1}$. But this is impossible, because there exists $x \in M_{\infty}$ with $|x|=1$; by $\Gamma$-symmetry all of the point $g(x)$ are in $M_{\infty}$, but these are not contained in any such sub-space.

Therefore we can apply Theorem 2 to the solution $\left\{M_{t}\right\}$, obtaining $C^{\infty}$ convergence to a limit $M_{T}$ satisfying the required identity (possibly after scaling to ensure $c=1) . M_{T}$ is $\Gamma$-symmetric, and has $\mathcal{Z}$ strictly less than that for the sphere solution, since $\mathcal{Z}$ has strictly decreased along the solution $\left\{M_{t}\right\}$. Therefore $M_{T}$ is non-spherical.

Corollary 20. In the case $n=1$, for each $k \geq 3$ and each $\alpha \in\left(0,1 /\left(k^{2}-\right.\right.$ 1)) there exists a non-circular strictly convex smooth curve $\mathcal{C}_{k}$ with $k$-fold symmetry which contracts homothetically under the flow

$$
\frac{\partial}{\partial t} x=-\kappa^{\alpha} \mathbf{n}
$$

where $\kappa$ is the curvature, and $\mathbf{n}$ the unit normal. 
Proof. For $k \geq 3$ the subgroup $\Gamma_{k}$ generated by rotation through $2 \pi / k$ satisfies the conditions of Theorem 18. The first spherical harmonics symmetric under $\Gamma_{k}$ are $\cos (k \theta)$ and $\sin (k \theta)$, with corresponding eigenvalue $\lambda=k^{2}$.

Corollary 21. In the case $n=2$, there exists a homothetically contracting solution of Eq. (6) with tetrahedral symmetry provided $\alpha \in(0,1 / 10)$; there exists one with octahedral symmetry provided $\alpha \in(0,1 / 18)$; and there exists one with icosahedral symmetry provided $\alpha \in(0,1 / 40)$.

Proof. In this case the only subgroups satisfying the required condition are the symmetry groups of the platonic solids. There are three such groups, since the dual solids have the same symmetry group. The first tetrahedrally symmetric spherical harmonic is given by the restriction of the function $x y z$ to $S^{2}$, and the corresponding eigenvalue is 12 . The first octahedrally symmetric spherical harmonic is $x^{4}+y^{4}+z^{4}-3 x^{2} y^{2}-3 x^{2} z^{2}-3 y^{2} z^{2}$, and the corresponding eigenvalue is 20 . Finally, the first icosahedrally symmetric spherical harmonic is

$$
\begin{aligned}
231 z^{6}-315 z^{4}\left(x^{2}+y^{2}\right)+105 z^{2}\left(x^{2}+y^{2}\right)^{2}-5\left(x^{2}+y^{2}\right)^{3} \\
+42 z x^{5}-420 z x^{3} y^{2}+210 z x y^{4},
\end{aligned}
$$

and the corresponding eigenvalue is 42 .

Corollary 22. For $n \geq 3$, there exists a non-spherical homothetically contracting solution with the symmetry of a regular $(n+2)$-simplex for $\alpha \in$ $(0,1 /(2(n+3)))$, and one with the symmetries of a regular hypercube for $\alpha \in(0,1 / 3(n+4))$.

Proof. The function $\sum_{i} x_{i}^{4}-(6 / n) \sum_{i \neq j} x_{i}^{2} x_{j}^{2}$ has the symmetry of a regular hypercube in $\mathbb{R}^{n+1}$, and its restriction to $S^{n}$ is a spherical harmonic with eigenvalue $4(n+3)$.

For all $n \geq 1$ there exists a cubic homogeneous harmonic polynomial $u_{n}$ on $\mathbb{R}^{n+1}$ with the symmetries of a regular simplex. These are given by the recursive definitions

$$
\begin{aligned}
u_{1}\left(x_{1}, x_{2}\right)= & x_{2}^{3}-3 x_{1}^{2} x_{2} \\
u_{n+1}\left(x_{1}, \ldots, x_{n+2}\right)= & u_{n}\left(x_{1}, \ldots, x_{n+1}\right) \\
& +\beta_{n}\left(x_{n+2}^{3}-\frac{3}{n+1}\left(x_{1}^{2}+\cdots+x_{n+1}^{2}\right)\right)
\end{aligned}
$$

where $\beta_{1}=\sqrt{2}$ and

$$
\beta_{k+1}=\beta_{k} \sqrt{\frac{(k+1)^{3}}{k^{2}(k+3)}} .
$$

The restriction of $u_{n}$ to $S^{n}$ is a spherical harmonic with the required symmetries, and the corresponding eigenvalue is $3(n+2)$. 
In [A5] we prove that the only homothetically contracting solutions of flows by positive powers of curvature are those given in Corollary 20. For $n=2$ we expect that there are many more solutions which are not described by Corollary 21. In particular, for small $\alpha$ there should be many solutions symmetric under each of the platonic symmetry groups, and there should also be solutions symmetric under some other subgroups of $S O(3)$, such as the subgroup of rotations about a fixed axis, and its discrete subgroups. In the case $n \geq 3$ there are of course many more examples of suitable subgroups $\Gamma$ which we have not mentioned explicitly in Corollary 22.

\section{Hypersurfaces with planar or cylindrical parts.}

In this section we demonstrate that the result of Theorem 1 no longer holds for any $\alpha>1 / n$. Specifically, we show that any solution starting from a hypersurface containing a planar region must still contain a planar region for small positive times. We also consider the behaviour of hypersurfaces which contain regions which are cylindrical or locally have the form $M^{n-k} \times \mathbb{R}^{k}$ for some $k>0$.

Theorem 23. Suppose $M_{0}$ is a compact convex hypersurface, and $F_{0}$ a subset of $M_{0}$ which has the form $N_{0}^{n-k} \times U_{0}^{k}$, where $N_{0}$ is a smooth convex hypersurface in $\mathbb{R}^{n+1-k}$ and $U_{0}$ is an open subset of $\mathbb{R}^{k}$. Then for any smooth positive function $\rho$ on $S^{n}$ and any $\alpha>1 / k$, the viscosity solution $\left\{M_{t}\right\}$ starting from $M_{0}$ contains an open subset $F_{t}$ of $F_{0}$ for $t>0$ sufficiently small.

Conversely, suppose $\Omega_{0}$ in a bounded open convex set in $\mathbb{R}^{n+1}$ for which

$$
\sigma_{n-k}=\inf _{x \in \partial \Omega_{0}} \sup _{P, \Gamma}\left\{\kappa: y \in \Omega_{0} \Rightarrow\left\langle x-y, P^{\perp}\right\rangle \geq \frac{1}{2} \kappa\left|\pi_{\Gamma}(x-y)\right|^{2}\right\}>0
$$

where the supremum is over all supporting hyperplanes $P$ of $\Omega_{0}$ which contain $x$ and all $n-k$-dimensional affine subspaces $\Gamma$ of $P$ through $x$, and $P^{\perp}$ is the unit normal to $P$ which points outward from $\Omega_{0}$. Then for any $\rho$ and any $\alpha \in(0,1 / k]$ the viscosity solution $\left\{\Omega_{t}\right\}$ of $(6)$ with initial condition $\Omega_{0}$ is smooth and strictly convex for $t>0$ and remains so until it contracts to a point.

When $\Omega_{0}$ is smooth, $\sigma_{n-k}$ is strictly positive if and only if $E_{n-k}(\mathcal{W})$ is strictly positive, or equivalently if and only if the sum of the smallest $k+1$ principal curvatures is strictly positive at every point.

Proof. To prove the first part of the Theorem, we will construct barriers with cylindrical symmetry, described by embeddings of the form $\varphi: S^{n-k} \times B_{R}^{k}(0)$ with $\varphi(z, x)=(u(|x|) z, x) \in \mathbb{R}^{n+1-k} \times \mathbb{R}^{k} \simeq \mathbb{R}^{n+1}$. We consider the case where $u$ is concave and decreasing in $|x|$. The metric and second fundamental 
form are given by

$$
\begin{aligned}
& g_{z_{i} z_{j}}=u^{2} \bar{g}_{i j} \\
& g_{x_{i} z_{j}}=0 \\
& g_{x_{i} x_{j}}=\delta_{i j}+\frac{x_{i} x_{j}}{|x|^{2}}\left(u^{\prime}\right)^{2}
\end{aligned}
$$

and

$$
\begin{aligned}
\mathcal{W}_{z_{i}}^{z_{j}} & =\frac{\delta_{i}^{j}}{u \sqrt{1+\left(u^{\prime}\right)^{2}}} \\
\mathcal{W}_{z_{i}}^{x_{j}} & =0 \\
\mathcal{W}_{x_{i}}^{x_{j}} & =-\frac{u^{\prime \prime}}{\left(1+\left(u^{\prime}\right)^{2}\right)^{3 / 2}} \frac{x_{i} x^{j}}{|x|^{2}}-\frac{u^{\prime}}{|x| \sqrt{1+\left(u^{\prime}\right)^{2}}}\left(\delta_{i}^{j}-\frac{x_{i} x^{j}}{|x|^{2}}\right) .
\end{aligned}
$$

The Gauss curvature is given by the expression

$$
K=(-1)^{k} \frac{u^{\prime \prime}\left(u^{\prime}\right)^{k-1}}{u^{n-k}|x|^{k-1}\left(1+\left(u^{\prime}\right)^{2}\right)^{(n+2) / 2}}
$$

and the unit normal is

$$
\nu=\frac{z-u^{\prime} \hat{x}}{\sqrt{1+\left(u^{\prime}\right)^{2}}}
$$

where $\hat{x}=x /|x|$.

It follows that any function $u(r, t)$ satisfying the inequality

$$
\dot{u} \leq-\sup \rho\left(-u^{\prime \prime}\right)^{\alpha}\left(-u^{\prime}\right)^{\alpha(k-1)} u^{-\alpha(n-k)}|x|^{-\alpha(k-1)}\left(1+\left(u^{\prime}\right)^{2}\right)^{\frac{1-\alpha(n+2)}{2}}
$$

has a cylindrical graph which acts as an inner barrier for convex solutions of Eq. (6). We proceed to construct such barriers.

Lemma 24. For given $k, \rho$, and $\alpha>1 / k$ there exist constants $c_{1}, c_{2}$ and $c_{3}$ such that for any $\lambda>0$ and $u_{0}>0, R>0$, the function

$$
u=u_{0}\left(1-c_{1}(k, \alpha)\left(\frac{|x|}{R}+c_{2}(\rho, k, \alpha)\left(\frac{u_{0}}{R}\right)^{2 k \alpha} \frac{t}{u_{0}^{1+n \alpha}}-1\right)_{+}^{\frac{k+1-1 / \alpha}{k-1 / \alpha}}\right)
$$

is $C^{2}$ and satisfies (25) on the region

$$
|x| \leq 2 R, \quad 0 \leq t \leq c_{3}(\rho, k, \alpha) u_{0}^{1+n \alpha}\left(\frac{R}{u_{0}}\right)^{2 k \alpha},
$$

provided $\alpha \in(1 / k, 1 /(k-1))$. If $\alpha \geq 1 /(k-1)$ then $u$ is not $C^{2}$ but acts as a barrier for smooth, strictly convex solutions of Eq. (6), hence also for the viscosity solutions constructed in Theorem 15. 
Proof. The case $\alpha \in(1 / k, 1 /(k-1))$ follows by direct computation. In the case $\alpha \geq 1 /(k-1)$ the same computation gives the result except when $|x|=R-c_{2}\left(u_{0} / R\right)^{2 k \alpha} R / u_{0}^{1+n \alpha} t$, where $u$ is not $C^{2}$. A smooth convex hypersurface lying outside the graph of $u$ and meeting the graph at such a point must have $K=0$, so the barrier condition is verified.

To use these barriers in the comparison principle, we need to check that the viscosity solution $\left\{M_{t}\right\}$ stays away from the boundary of the barrier produced in Lemma 24. Fix $x$ in the interior of $M_{0}$ and $\varepsilon>0$ small, and choose $u_{0}$ smaller than the smallest radius of curvature of $N_{0}$, and place the origin at the point $x-\left(u_{0}+\varepsilon\right) \nu_{x}$. Choose $R$ sufficiently small so that the distance from $x$ to the boundary of $F_{0}$ is at least $3 R$. Let $\tilde{M}_{0}$ be the hypersurface given by

$$
\sqrt{\sum_{i=1}^{n-k} x_{i}^{2}}=u_{0}\left(1-c_{1}(k, \alpha)\left(\frac{\sqrt{\sum_{i=n-k+1}^{n} x_{i}^{2}}}{R}-1\right)_{+}^{\frac{k+1-1 / \alpha}{k-1 / \alpha}}\right)
$$

for $\sum_{i=n-k+1}^{n} x_{i}^{2} \leq 4 R^{2}$. Then $\tilde{M}_{0}$ lies entirely within $M_{0}$. Furthermore, for each $y \in \partial \tilde{M}_{0}$, the sphere $B_{c(\rho, k, \alpha) \min \left\{u_{0}, R\right\}}(y)$ is enclosed by $M_{0}$ for some constant $c$. Define $\tilde{M}_{t}$ to be the hypersurface

$\sqrt{\sum_{i=1}^{n-k} x_{i}^{2}}=u_{0}\left(1-c_{1}\left(\frac{\sqrt{\sum_{i=n-k+1}^{n} x_{i}^{2}}}{R}+c_{2}\left(\frac{u_{0}}{R}\right)^{2 k \alpha} \frac{t}{u_{0}^{1+n \alpha}}-1\right)_{+}^{\frac{k+1-1 / \alpha}{k-1 / \alpha}}\right)$ for $\sum_{i=n-k+1}^{n} x_{i}^{2} \leq 4 R^{2}$. For $t<c(\rho, n, k, \alpha) \min \left\{u_{0}, R\right\}^{1+n \alpha}$ we have

$$
\partial \tilde{M}_{t} \subset \bigcup_{y \in \partial \tilde{M}_{0}} B_{r(t)}(y)
$$

where $r(t)^{1+n \alpha}=\left(c \min \left\{u_{0}, R\right\}\right)^{1+n \alpha}-(1+n \alpha) \sup \rho t$. The comparison principle implies that each of these balls is enclosed by $M_{t}$, and therefore that $\partial \tilde{M}_{t}$ does not meet $M_{t}$. It follows by the comparison principle that $\tilde{M}_{t}$ remains entirely enclosed by $M_{t}$ on this time interval, and therefore that $h\left(\nu_{x}, t\right) \geq h\left(\nu_{x}, t\right)-\varepsilon$ for all $\varepsilon>0$, so that $x$ has not moved during this time interval.

Now we proceed to the second part of the Theorem, the case of $\alpha \leq 1 / k$. This also proceeds using barrier constructions. Let $x \in \partial \Omega_{0}$. Since $\sigma_{n-k}>0$, there exists a hyperplane $P$ supporting $\Omega_{0}$ at $x$ and an $n-k$-dimensional subspace $\Gamma$ of $P$ such that $\Omega_{0}$ is contained in the region

$$
\left\langle x-y, P^{\perp}\right\rangle \geq \frac{1}{4} \sigma_{n-k}\left|\pi_{\Gamma}(x-y)\right|^{2} .
$$


$\Omega_{0}$ is also clearly contained in the ball $B_{2 r_{+}\left(\Omega_{0}\right)}(x)$. We now construct barriers:

Lemma 25. Fix $k, \rho, \alpha \in(0,1 / k)$ and constants $R>0$ and $u_{0}>0$. If every $y \in \Omega_{0}$ satisfies

$$
|y| \leq R, \sum_{i=1}^{n-k} y_{i}^{2} \leq u_{0}^{2}
$$

then for $0<t \leq c(\rho, k, n, \alpha) \min \left\{u_{0}, R\right\}^{1+n \alpha}$ we have for every $y=(Y, \eta) \in$ $\Omega_{t} \subset \mathbb{R}^{n+1-k} \times \mathbb{R}^{k} \simeq \mathbb{R}^{n+1}$,

$$
|Y| \leq u_{0}-c t^{\frac{1}{1-k \alpha}} \frac{R^{\frac{\alpha-1}{1-k \alpha}}}{u_{0}^{\frac{\alpha(n-k)}{1-k \alpha}}}\left((4 R-|\eta|)^{-\frac{\alpha(k+1)}{1-k \alpha}}+(4 R+|\eta|)^{-\frac{\alpha(k+1)}{1-k \alpha}}\right)
$$

where $c$ depends only on $\rho, k, n$, and $\alpha$. If $\alpha=1 / k$ and every $y \in \Omega_{0}$ satisfies (26), then for $0<t \leq c(\rho, k, n) \min \left\{u_{0}, R\right\}^{1+n / k}$ and $y=(Y, \eta) \in \Omega_{t}$ we have

$$
|Y| \leq u_{0}-c_{1} R \exp \left(-c_{2} R^{2 k} u_{0}^{n-k} t^{-k}\right) \cosh \left(c_{2} R^{2 k-1} u_{0}^{n-k}|\eta| t^{-k}\right)
$$

where $c_{1}$ and $c_{2}$ depend only on $\rho, k$, and $n$.

Proof. A direct calculation shows that these regions are given by cylindrical graphs satisfying the inequality

$$
\dot{u} \geq-\inf \rho\left(-u^{\prime \prime}\right)^{\alpha}\left(-u^{\prime}\right)^{\alpha(k-1)} u^{-\alpha(n-k)}|x|^{-\alpha(k-1)}\left(1+\left(u^{\prime}\right)^{2}\right)^{\frac{1-\alpha(n+2)}{2}}
$$

which therefore act as outer barriers for solutions of Eq. (6).

It follows that the support function at every point must change: If we fix $z \in S^{n}$, and place a cylindrical barrier outside the hypersurface $M_{0}$ and passing through $x$, then we have

$$
h(z, t) \leq h(z, 0)-c t^{\frac{1}{1-k \alpha}} R^{-\frac{1+k \alpha}{1-k \alpha}} u_{0}^{-\frac{\alpha(n-k)}{1-k \alpha}}
$$

for $\alpha<1 / k$, and

$$
h(z, t) \leq h(z, 0)-c_{1} R \exp \left(-c_{2} R^{2 k} u_{0}^{n-k} t^{-k}\right)
$$

when $\alpha=1 / k$. Theorem 7 then gives lower bounds on the speed $\rho S_{n}^{-\alpha}$ uniformly in $z$ for each $t>0$, by the same argument as given in the proof of Theorem 8. Theorem 10 implies bounds below on all the principal curvatures at each positive time, and Corollary 12 gives upper bounds on the principal curvatures. Theorem 5.5 of $[\mathbf{K}]$ implies that the solution hypersurface is smooth and strictly convex for sufficiently small positive times, and the result of Theorem 23 follows from Theorem 13. 


\section{References}

[AGLM] L. Alvarez, F. Guichard, P.L. Lions and J.M. Morel, Axioms and fundamental equations of image processing, Arch. Rat. Mech. Anal., 123 (1993), 199-257.

[A1] B. Andrews, Contraction of convex hypersurfaces in Euclidean space, Calc. Var. \& P.D.E., 2 (1994), 151-171.

[A2] Evolving convex curves, Calculus of Variations and P.D.E., 7 (1998), 315-371.

[A3] Entropy estimates for evolving hypersurfaces, Comm. Analysis and Geometry, 2 (1994), 53-64.

[A4] Contraction of convex hypersurfaces by their affine normal, J. Differential Geometry, 43 (1996), 207-230.

[A5] _ Classification of limiting shapes for isotropic curve flows, preprint in preparation.

[A6] Monotone quantities and unique limits for evolving convex hypersurfaces, Int. Math. Res. Not., (1997), 1001-1031.

[A7] _ Harnack inequalitites for evolving hypersurfaces, Math. Z., 217 (1994), 179-197.

[A8] - Instability of limiting shapes for curves evolving by curvature, preprint.

[A9] - Instability and non-convergence in Gauss curvature flows, preprint in preparation.

[A10] The affine curve-lengthening flow, Journal für die reine und angewandte Mathematik, 506 (1999), 43-83.

[AST] S. Angenent, G. Sapiro \& A. Tannenbaum, On the affine heat equation for nonconvex curves, preprint, 1997 (37 pages).

[B] W. Blaschke, Vorlesungen über Differentialgeometrie II, Verlag von Julius Springer, Berlin, 1923.

[Ca] E. Calabi, Complete affine hyperspheres I, Ist. NAZ Alta Mat. Sym.Mat., X (1972), 19-38.

[CS] V. Caselles and C. Sbert, What is the best causal scale-space for $3 D$ images?, SIAM J. Appl. Math., 56 (1996), 1199-1246.

[Ch1] B. Chow, Deforming convex hypersurfaces by the nth root of the Gaussian curvature, J. Differential Geometry, 22 (1985), 117-138.

[Ch2] - Deforming hypersurfaces by the square root of the scalar curvature, Invent. Math., 87 (1987), 63-82.

[Ch3] On Harnack's inequality and entropy for the Gaussian curvature flow, Comm. Pure Appl. Math., 44 (1991), 469-483.

[Fi] W.J. Firey, On the shapes of worn stones, Mathematika, 21 (1974), 1-11.

[Ga1] M.E. Gage, An isoperimetric inequality with applications to curve shortening, Duke Math. J., 50 (1983), 1225-1229.

[Ga2] _ Curve shortening makes convex curves circular, Invent. Math., 76 (1984), 357-364.

[Ga3] Evolving plane curves by curvature in relative geometries, Duke Math. J., 72 (1993), 441-466. 
[GH] M.E. Gage and R.S. Hamilton, The heat equation shrinking convex plane curves, J. Differential Geometry, 23 (1986), 69-96.

[Gr] M. Grayson, The heat equation shrinks embedded closed curves to round points, J. Differential Geometry, 26 (1987), 285-314.

[Ha1] R. Hamilton, Worn stones with flat sides, in 'A tribute to Ilya Bakelman' (College Station, TX 1993), 69-78; Discourses Math. Appl., 3, Texas A\&M Univ., College Station, TX, 1994.

[Ha2] Remarks on the entropy and Harnack estimates for the Gauss curvature flow, Comm. Anal. Geom., 2 (1994), 155-165.

[Hu] G. Huisken, Contraction of convex hypersurfaces by their mean curvature, J. Differential Geometry, 20 (1984), 237-268.

[K] N.V. Krylov, Nonlinear elliptic and parabolic equations of second order, D. Reidel, 1987.

[Le] K. Leichtweiss, On inner parallel bodies in the equiaffine geometry, Analysis and geometry (Eds. B. Fuchssteiner and W.A.J. Luxemburg), BI-Verlag, Mannheim, 1992, 113-123.

[Li] G. M. Lieberman, Second order parabolic differential equations, World Scientific, Singapore, 1996.

[Lu] A. Lunardi, Analytic Semigroups and Optimal Regularity in Parabolic Problems, Birk-häuser, Basel, 1995.

[Mi] D.S. Mitrinowic, Analytic inequalities, Springer-Verlag, Berlin-Heidelberg-New York, 1970.

[NK] P. Neskovic and B. Kimia, Three-dimensional shape representation from curvature-dependent surface evolution, Technical report LEMS-128, Divison of Engineering, Brown University, 1993.

[Oa] J. Oaks, Singularities and self-intersections of curves evolving on surfaces, Indiana Univ. Math. J., 43 (1994), 959-981.

[OST] P. Olver, G. Sapiro and A. Tannenbaum, Invariant geometric evolutions of surfaces and volumetric smoothing, SIAM J. Appl. Math., 57 (1997), 176-194.

[Sa] L. Santaló, Un invariante afin para los cuerpos convexos del espacio de $n$ dimensiones, Portugal. Math., 8 (1949), 155-161.

[ST1] G. Sapiro and A. Tannenbaum, On invariant curve evolution and image analysis, Indiana Univ. Math. J., 42 (1993), 985-1009.

[ST2] _ Affine invariant scale-spaces, Internat. J. Comput. Vision, 11 (1993), $25-44$.

[ST3] _ On affine plane curve evolution, J. Funct. Anal., 119 (1994), 79-120.

[Sc] R. Schneider, Convex bodies: The Brunn-Minkowski Theory, Encyclopedia of Mathematics, 44, Cambridge University Press, 1993.

[Si] J. Simons, Minimal varieties in Riemannian manifolds, Ann. Math., 88 (1968), 62-105.

[Ts] K. Tso, Deforming a hypersurface by its Gauss-Kronecker curvature, Comm. Pure Appl. Math., 38 (1985), 867-882.

[U1] J.I.E. Urbas, An expansion of convex surfaces, J. Differential Geometry, 33 (1991), 91-125. 
$[\mathrm{U} 2]$ Complete noncompact self-similar solutions of Gauss curvature flows I. Positive powers, Math. Ann., 311 (1998), 251-274.

Received October 28, 1998. This research was partially supported by an Australian Research Council QEII Fellowship.

Australian National University

A.C.T. 0200

Australia

E-mail address: andrews@maths.anu.edu.au 